\title{
Underwater pre-touch based on artificial electric sense
}

\author{
Frédéric Boyer, Vincent Lebastard*, Steven Bruce Ferrer ${ }^{\dagger}$ and Franck Geffard ${ }^{\dagger} \ddagger$
}

December 9, 2019

\begin{abstract}
This article exploits a bio-inspired sensor technology named artificial electric-sense to emulate underwater pre-touch. The sensor is considered as an electric finger remote-controlled by an operator to follow the boundaries of objects. Using electric measurements only, the approach feeds back pre-touch forces and torques to the operator through an haptic interface. These forces and torques are generated by a set of virtual electric charges and dipoles arranged on the probe and reacting in the electric field reflected by the objects. This model of emulated forces is passive and guarantees the stability of a position-position haptic feedback loop. The whole approach is assessed through a set of experiments carried out on a Cartesian slave robot coupled to an haptic interface. The obtained results show the feasibility of the concept and its robustness to different configuration of objects. Such an electro-haptic feedback opens new perspectives in both electric field sensing and underwater robotics.
\end{abstract}

\section{Introduction}

Discovered in the 50s by Lissman and Machin (Lissmann \& Machin 1958), active electric sense or "e-sense", is a sensorial ability evolved by several hundreds of fresh water fish species, which perceive their environment by measuring the perturbations of a self-generated electric field. Weakly electric fish as Gnathonemus Petersii, use electric sense to navigate turbid waters with many obstacles, communicate between con-specifics, find their preys and escape their predators (Von der Emde \& Bleckmann 1998), (Caputi et al. 1998). The same fish are able to sense exogenous electric fields generated by other fish, another modality of electric sense named passive electric sense (Hopkins 2005). Remarkably, passive electric sense is also used by several species of marine fish, as the shark Scyliorhinus canicula or the ray Raja clavata for finding their preys burrowed in the sand (Kalmijn 1971). In the recent past years this sense has attracted the interest of robotics with the aim of equipping a new generation of underwater robots able to operate in confined spaces

\footnotetext{
${ }^{*} \mathrm{~V}$. Lebastard and F. Boyer are with the LS2N Laboratory, Institut Mines Telecom Atlantique, 44307 Nantes, France, vincent.lebastard@mines-nantes.fr frederic.boyer@mines-nantes.fr

${ }^{\dagger}$ B. Ferrer and F. Geffard are resp. with, CEA Tech Pays de la Loire, Technocampus, F-44340 Bouguenais, France, and with CEA, LIST, SRI, F-91120 Palaiseau, France, steven.ferrer@cea.fr, franck.geffard@cea.fr, steven.ferrer@cea.fr franck.geffard@cea.fr

† The work presented was performed as part of the strategic partnership between CEA Tech, Mines Nantes and ARMINES, funded by the region of Pays de La Loire, in the frame of the Regional Technology Transfer Platform (PRTT) of CEA Tech.
} 
with muddy waters (Solberg et al. 2008), (Silverman et al. 2012), (Boyer et al. 2015), (Lebastard et al. 2016). In this perspective, several electric sensors have been proposed and can be classified in two major categories depending whether the electric field is measured through floating voltages (Bai et al. 2012) or electric currents (Servagent et al. 2013). In (Servagent et al. 2013), the current measurement mode has been implemented through a family of sensors consisting of some plastic slender probes on which are arranged a few metal electrodes in different configurations (see one of them in figure 1-b). In recent years, all these sensor technologies were used to address different issues in underwater robotics ranging from model-based localization (Solberg et al. 2008, Lebastard et al. 2013), to reactive navigation (Boyer et al. 2013), underwater docking (Boyer et al. 2015), and shape recognition (Bai et al. 2015, Lanneau et al. 2017). Recently, the electric sense has been proposed as an assistance for visual feedback in virtual reality (Fang et al. 2016). In the present article, it is also used in a feedback loop to assist a human operator. However, in contrast to (Fang et al. 2016), the assistance is not based on visual feedback and virtual reality, but exploits the haptic modality of e-sense (Caputi et al. 2013) with the sensor of (Servagent et al. 2013), to emulate underwater pre-touch. As a first illustration of this concept, the article shows how an operator can use an electric probe as an electric finger, moving it along the boundaries of objects at a given distance, while having the feeling of touching their surface (see figure 1-a-left). To address this task, we will face the following fundamental issue. Let us consider a slave manipulator remote-controlled by a master haptic interface. The slave is equipped with an active probe at its tip. The probe is immersed in water in presence of an infinite wall. Knowing the electric currents measured by the probe and the configuration of the manipulator only, we want to construct a set of forces which once fed back to the operator through the haptic interface, gives him the feeling that: (1) In a direction parallel to the wall, the probe can be moved with no effort. (2) Along other DoFs, some restoring forces appear as soon as the probe is no longer perpendicular to the wall at a prescribed distance $d$ from it (see figure 1-a-right).

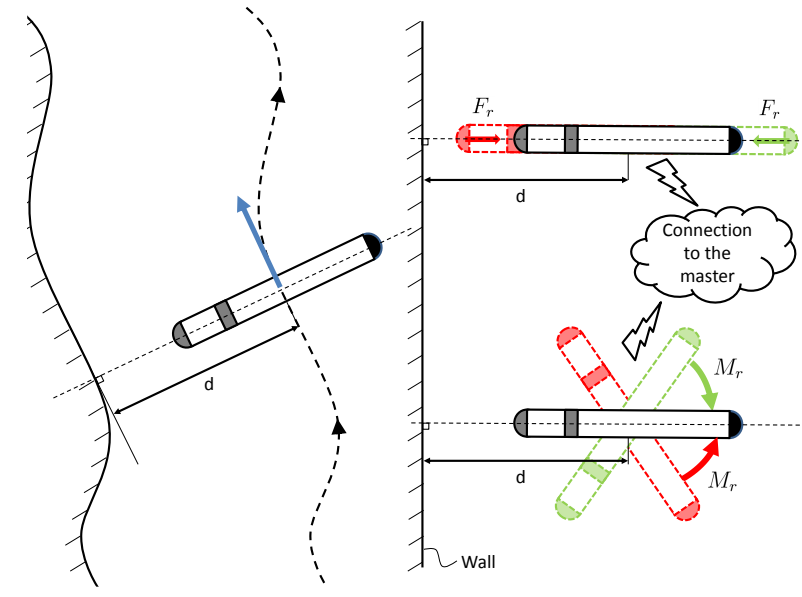

(a)

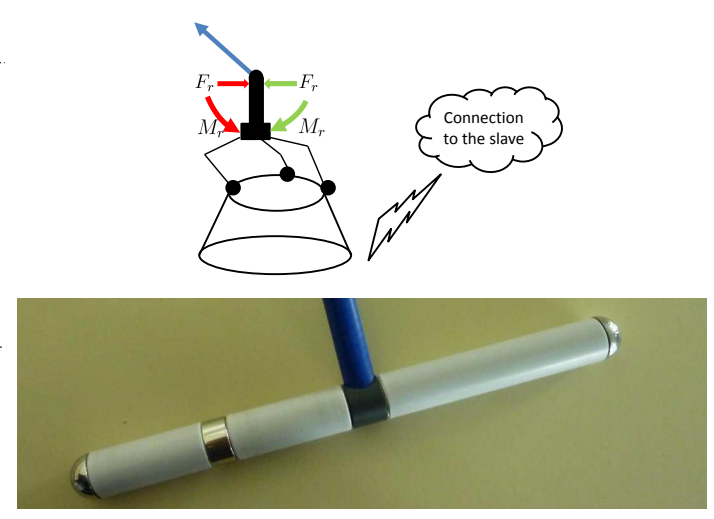

(b)

Figure 1: Schematize of the targeted scenario with restoring electric force and torque $F_{r}$ and $M_{r}$ (green and red arrows) and free-force direction (blue arrows) in slave (a), and master (b-top) spaces. Picture of a 3-electrode probe (b-bottom).

This issue could be addressed by electro-locating the probe in a virtual environment, or "proxy", and by providing the operator with an haptic feedback based on this geometric model (Mitra \& Niemeyer 2004). However, in realistic conditions, the geometric reconstruction of such a proxy 
would require addressing complex inverse problems which remain largely unsolved so far. To circumvent this difficulty, we here privileged a sensor-based approach which avoids resorting to such a virtual model. In this approach, the forces and torques exerted onto the probe, are generated by a set of virtual electric charges and dipoles arranged along its axis, which reacts in the electric field reflected by its environment. Designing the Coulomb charges and dipoles accordingly, the final model stands for a generalization of the so-called Lennard-Johns attractive-repulsive forces used in physics of gases and liquids to model the interactions between atoms (Hirschfelder et al. 1966). Though conceptually developed for an infinite wall, we will see how this approach remains robust in more realistic experimental conditions including perturbations induced by corners and objects.

The contribution of this article is two folds. First, it shows how the haptic modality of electric sense can be exploited to implement the first 3D electro-haptic remote control loop. In this context, by combining several concepts in electrostatics and remote control, it can open new perspectives for electric field sensing in general. Second, it proposes a new sensorial feedback modality which could assist teleoperated underwater robotics arms on Remotly Operated Vehicles in harsh conditions as in the case of dam inspection (Maalouf et al. 2012).

The subsequent developments are structured as follows. In section 2 , the experimental context of the article is presented. This includes the electric probe and test bed as well as the haptic interface. The next section (3) briefly reminds the basic concepts of haptic feedback control used in the article. In particular, the stability of a feedback-control loop is here considered from the view point of passivity. Based on this general concept, in section 4, a definition of a passive restoring force is proposed and illustrated in section 5, on a simple, but instructive example (two charged particles remote-controlled along one dimension). We then start to address our general problem, and present in section 6 the model of the environment including the geometry of the scene, the model of the electric field reflected by a wall, the model of the electric measurements of a probe, and finally, the sensor-based model of the electric field along the probe. Based on this latter model, the article continues with its theoretical core which consists of designing a model of the electric forces exerted onto the probe (section 7). In section 8, an experimental implementation of the model is proposed. In section 9 we introduce the position-position architecture of the haptic feedback loop. The entire approach is experimented in section 10, which starts with its characterization through some preliminary tests, and pursues with operational tests in section 11. Finally, the article is closed by a conclusion which opens further perspectives for future (section 12).

\section{Experimental context}

A force-feedback (or bilateral) remote-control loop is composed of five components: a human operator, a master manipulator, a slave manipulator, and a coupling controller between both manipulators and the environment. The sub-system "master-coupling-slave" is named a Teleoperation System (TS). Once included into the loop, the operator exchanges forces, positions and velocities with the master, and wishes to have the feeling of interacting directly with the environment. In most industrial applications the master and the slave arms are coupled by some mechanical devices (e.g. steal wires) (Coiffet \& Vertut 1985), and the environment is constituted of real objects more or less stiff and heavy. In our particular case, the coupling is realized by a 
computer (Geffard et al. 2012), and the environment consists of a given scene in interaction with an active electric sensor through some emulated forces that remains to be defined (see figure 2).

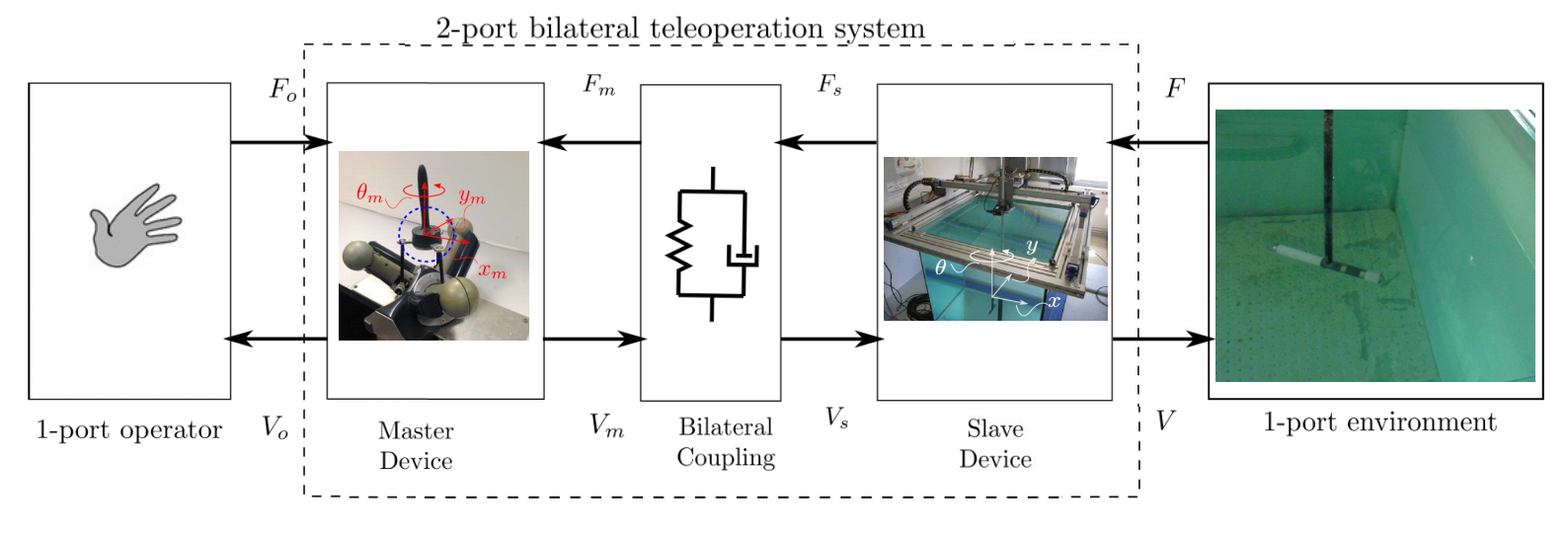

Figure 2: Architecture of the remote control loop as a network of multiport blocks exchanging pairs of dual (force-velocity) variables $(F, V)$, with $o, m, s$ for "operational", "master" and "slave".

In the rest of this section, we present our experimental devices that compose the architecture of figure 2 .

\section{$2.1 \quad$ Electric probe}

Our electric sensors are slender probes constituted of a cylindrical insulating (plastic) shell on which are fixed several groups of metal electrodes in contact with water (Servagent et al. 2013). As represented in figure 1-b, we here consider a probe with 3 groups of electrodes, named, the head, neck and tail electrodes. The head and the neck electrodes are subdivided into left-right symmetrical sub-electrodes which provide an independent measurement of the electric currents that flow across each of them. This probe can be used with same electronics in passive and active modes, depending whether the electric currents it measures, are generated outside the probe or by the probe itself. In active mode, the head and neck electrodes are set under voltage with respect to the tail one, with a wave generator. Due to the Ohmic character of the water, this generates a field of currents flowing from the tail electrode, named the emitter, to the two others, named the receivers.

\section{$2.2 \quad$ Electric test-bed and slave manipulator}

Our experiments are carried out in a $1 \mathrm{~m}^{3}$ tank filled with ordinary tap water of conductivity $\simeq 0.04 \mathrm{~S} \cdot \mathrm{m}^{-1}$. The top of the tank is equipped with our slave manipulator. This is a 3-dof Cartesian robot (a gantry) that allows controlling the motions of a vertical carbon fiber rod at the tip of which is fixed the probe (see figure $3(\mathrm{a})$ ). The gantry allows translating the probe along the two horizontal axes $(x, y)$, as well as rotating it about the vertical yaw axis $(\theta)$. This is performed with maximum velocities $0.1 \mathrm{~m} \cdot \mathrm{s}^{-1}$ and $80^{\circ} \mathrm{s}^{-1}$, and with a precision of $1 / 10 \mathrm{~mm}$ and $0.023^{\circ}$. 


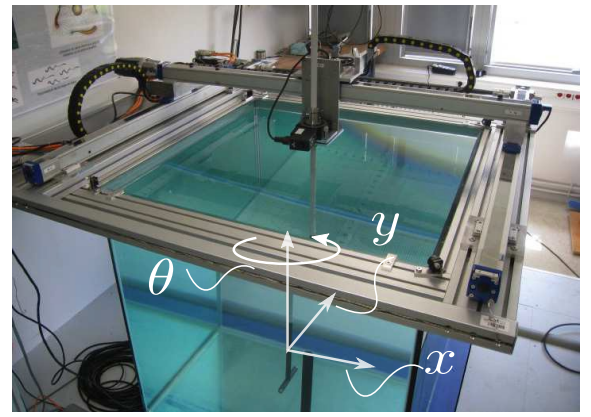

(a)

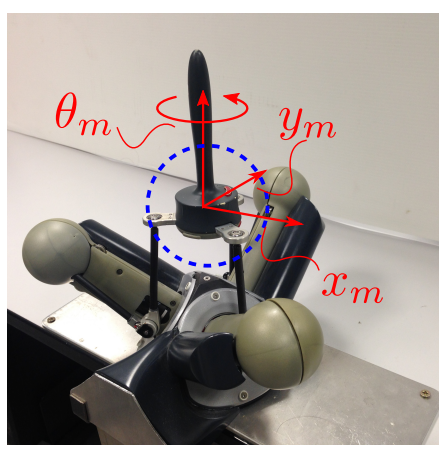

(b)

Figure 3: (a) Tank of $1 \mathrm{~m}^{3}$ with the gantry above. (b) Haptic interface 6D Desktop of Haption with its position control zone (blue sphere).

\subsection{Master manipulator}

The slave manipulator is remote controlled by the operator through an haptic display pictured in figure 3 (b). This is a 6 -DoF master parallel manipulator $(3 \times 2$ parallel haptic interface of Haption $^{\mathrm{TM}}$ ) whose design is detailed in (Gosselin et al. 2005). Its useful workspace consists of a $150 \mathrm{~mm}$ diameter sphere. The homogeneous orientation workspace remains over $\pm 30^{\circ}$ in a $100 \mathrm{~mm}$ diameter sphere. It can transmit maximum force and torque of $20 \mathrm{~N}$ and $0.5 \mathrm{Nm}$ in the centre of the workspace, and force and torque over $14.3 \mathrm{~N}$ and $0.32 \mathrm{Nm}$ in the $150 \mathrm{~mm}$ diameter spherical workspace. This haptic display is an impedance display, i.e., it measures position and displays force. It has been designed to be transparent (with low friction and inertia).

\subsection{Coupling controller}

A controller named TAO (Geffard et al. 2012), is used to perform the Cartesian coupling between the master and the slave spaces. This controller is a core software platform dedicated to Computer Aided force-feedback Teleoperation. It is able to control different types of manipulators. In our case, in spite of the obvious differences of kinematics between our master and slave manipulator, TAO ensures homothetic displacements in the two Cartesian spaces. It allows high speed synchronization between several real and virtual mechanisms (master arm, slave arm, camera, Virtual Reality or dynamic simulation engine to name but a few), and provides several control modes and operator assistances. It can be used to implement different types of coupling (position-position, force-position, position-force, 3 or 4 channels...). After this short presentation of the experimental context, the next section provides an intuitive introduction to the basic concepts of haptic feedback control which are used throughout the article.

\section{Characteristics of a haptic-feedback loop}

As above introduced, a TS is composed of sensors, amplifiers, motors, software which interact at one side with an operator, and at the other, with a real or a simulated environment. The operator and environment are both non-linear systems with varying dynamics and structure. Under these constraints, designing and tuning a TS able to guaranty the stability of the overall loop (including the operator and environment) is a challenging issue to which modelling and frequency approaches 
are difficult to apply. This is why, in conventional TS, one commonly assumes the passivity of the operator and even of the environment, a feature which greatly simplifies stability analysis (Hogan 1989). In this paper, we will resort to this concept to design our feedback loop. In particular, a particular attention will be paid to design a passive model of restoring electric forces.

\subsection{Transparency}

Intuitively, a remote-controlled system is perfectly transparent if the operator has the feeling of directly operating on the environment. Referring to the network representation of figure 2, this means that the relations between forces and velocities are identical in the slave and master spaces. In the linear case (i.e., if all the blocks of figure 2 can be modelled by transfer matrices), ideal transparency is thus achieved when we have $Z_{t}=Z_{e}$, where $Z_{t}=F_{o} / V_{o}$ and $Z_{e}=F / V$ are the transmission and the environment impedances (Hannaford 1989). Ensuring stability with perfect transparency is in general impossible. Thus, in practice, the global performance of a remote control loop is the result of a compromise between these two antagonist ideal goals.

\subsection{Stability and passivity}

Stability of haptic systems is intuitively satisfied when an operator can interact with the distant environment through an haptic interface without any parasitic oscillations. There are several ways to test stability of such a system. One of the most intuitive ones is to use the concept of passivity. It allows to guarantee the stability without an exact knowledge of the system model and parameter values. In short, a system is said passive, if the integral of the power extracted from it over time, does not exceed the initial energy stored in the system (Colgate \& Brown 1994). One important property when studying stability of haptic displays consists of the fact that the serial connection of two passive elements is also passive. In the subsequent developments, we will exploit this property to guarantee the stability of our electro-haptic loop. To that end, we will implement the passive unconditionally stable position-position TS architecture (Aliaga et al. 2004), and will design a model of the environment in the form of some restoring electric forces that fulfill the passivity property. Therefore, the passivity of all the components of figure 2 (including that of the operator that will be assumed), will then ensure the stability of the whole system. Before starting this design, we provide in the next section a definition of a restoring force in relation to the general concept of passivity.

\section{Concept of passive restoring force}

Let us consider a mechanical system with configuration parameters $X \in \mathcal{C}$. Intuitively, a restoring force $F_{r}(X)$ applied to this system is a force that opposes any $d X$ moving the system away from an equilibrium point $X_{d}$, i.e., such that:

$$
F_{r}\left(X_{d}\right)=0
$$

Such a force is passive if pushing the system out of $X_{d}$ along any path, always requires imparting a positive energy to it, i.e., if we have:

$$
-\int_{0}^{t} F_{r}^{T} V d \nu \geq 0, \forall \text { paths in } \mathcal{C},
$$


where $t$ is the time-variable, and $V$, the velocity of the system. Now assuming that $F_{r}$ is conservative, there exists a state function $U$ such that $F_{r}(X)=-\nabla_{X} U$, and (2) can be rewritten as:

$$
-\int_{0}^{t} F_{r}^{T} V d \nu=-\int_{X_{d}}^{X} F_{r}^{T} d Z=U(X)-U\left(X_{d}\right)=S(X) \geq 0, \forall X \in \mathcal{C} .
$$

In this condition, the configuration dependent function $S$ fulfills all the requirements of a storing (energy) function for the lossless passive force $F_{r}$. Finally, since $S\left(X_{d}\right)=0$, to ensure $F_{r}$ to be a passive restoring force, it suffices to fulfill the three following conditions:

1. $F_{r}$ is conservative.

2. The point $X_{d}$ is an equilibrium point, i.e., it is such that (1) holds.

3. The equilibrium point $X_{d}$ is the only minimum of the function $S($.$) defined by (3).$

In all the following, we will seek to build a model of electric forces that fulfills the above conditions. Before investigating this issue, we illustrate the above concept on a simple case which plays a key role in our application.

\section{Illustrative example: emulation of Lennard-Johns forces}

For the sake of illustration, we start by considering an idealized one-dimensional translational remote-controlled system (i.e. $\mathcal{C}=\mathbb{R}$ ). It consists of a set of two electric particles $P_{1}$ and $P_{2}$ of charge $q_{1}$ and $q_{2}$ respectively, both connected through a rigid line of length $l$ (see figure 4 ). This rigid system can move along the $x$-axis, and $P_{1}$ and $P_{2}$ have for abscissa $x_{1}=X-(l / 2)$ and $x_{2}=X+(l / 2)$, with $X \in \mathbb{R}$, the configuration parameter of this rigid system. In this simple case, our general objective consists of emulating a restoring force around a desired position $X_{d}$, the current position of the system $X$ being known through measurements. With no loss of generality we assume that $X, X_{d}>0$. This system is now immersed in a 1-dimensional electric field of the form

$$
E(x)=\frac{1}{x^{2}},
$$

where $x$ here represents the distance between any point along the $x$-axis and a unit charge particle located in $x=0$. According to laws of electrostatics (Jackson 1999), each of the two particles is subject to a 1-dimensional (conservative) Coulomb force that reads

$$
F_{i}=q_{i} E\left(x_{i}\right)=\frac{q_{i}}{x_{i}^{2}}, i=1,2
$$

From this simple physical picture, for any values of $q_{1}$ and $q_{2}$, one can build a restoring force $F_{r}$ around any position of equilibrium $x_{d}$ along the $x$-axis by using the simple model

$$
F_{r}(X)=F(X)-F_{d}=\left(\frac{q_{1}}{x_{1}^{2}}+\frac{q_{2}}{x_{2}^{2}}\right)-\left(\frac{q_{1}}{x_{1 d}^{2}}+\frac{q_{2}}{x_{2 d}^{2}}\right),
$$

where $F_{d}=F\left(X_{d}\right)$, and $X_{d}=x_{1 d}+l / 2=x_{2 d}-l / 2$ denotes the desired position of the system as this is illustrated by the schematize of figure 4 . Now, let us remark that choosing the two charges such that

$$
\frac{q_{2}}{q_{1}}=-\left(\frac{x_{2 d}}{x_{1 d}}\right)^{2},
$$




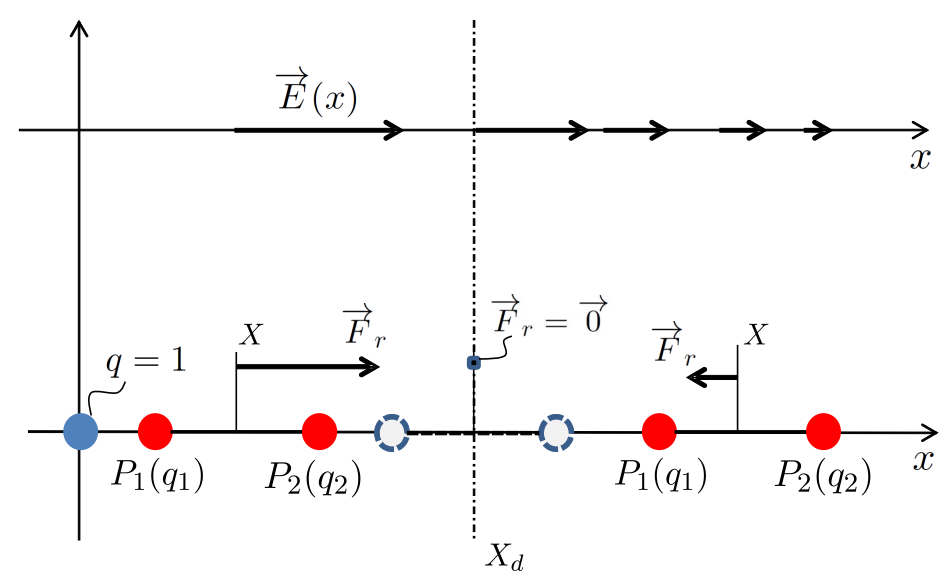

Figure 4: Emulation of a restoring force with two Coulomb charges immersed in an one-dimensional electric field. Top axis: electric field. Bottom axis: Restoring force.

with $q_{1}>0$, allows generating a zero resultant force $F_{d}$ in the desired position. In this case, the restoring force (6) is reduced to

$$
F_{r}(X)=F(X)=q_{1}\left(\left(\frac{1}{x_{1}}\right)^{2}-\left(\frac{x_{2 d}}{x_{1 d}}\right)^{2}\left(\frac{1}{x_{2}}\right)^{2}\right),
$$

or again, in terms of the dimensionless variables $\eta=2 X / l, \eta_{d}=2 X_{d} / l$ and $\xi=\eta-\eta_{d}$ :

$$
F_{r}(\xi)=\frac{4 q_{1}}{l^{2}}\left(\left(\frac{1}{\xi+\eta_{d}-1}\right)^{2}-\left(\frac{\eta_{d}+1}{\eta_{d}-1}\right)^{2}\left(\frac{1}{\xi+\eta_{d}+1}\right)^{2}\right) .
$$

Then, inserting the expression (8) in the path integral of (3) gives:

$$
S(X)=-\int_{0}^{t} F_{r} \dot{X} d \nu=-q_{1} \int_{x_{1 d}}^{x_{1}} \frac{d z_{1}}{z_{1}^{2}}+q_{1}\left(\frac{x_{2 d}}{x_{1 d}}\right)^{2} \int_{x_{2 d}}^{x_{2}} \frac{d z_{2}}{z_{2}^{2}} .
$$

Now using (9), and because Coulomb forces are conservative ${ }^{1},(10)$ can be integrated as

$$
S(\xi)=\frac{2 q_{1}}{l}\left[\left(\frac{1}{\xi+\eta_{d}-1}-\frac{1}{\eta_{d}-1}\right)-\left(\frac{\eta_{d}+1}{\eta_{d}-1}\right)^{2}\left(\frac{1}{\xi+\eta_{d}+1}-\frac{1}{\eta_{d}+1}\right)\right] .
$$

Finally, for any $X_{d}$ and any positive number $q_{1}$, the right hand side of (11) defines a nonnegative storing function $S(\xi)$. This context is illustrated in figure 5 which displays several plots of $F_{r}(\xi)$ and $S(\xi)$ for $q_{1}=1, l=0.03$ and different values of $\eta_{d}=2 X_{d} / l$. It is straightforward to show that this model fulfills the three conditions at the end of section 4. Therefore, it is unconditionally passive on the half positive axis. Moreover, the plots of figure 5 have the typical shape of the Lennard-Johns force and potential, which model the competition between long range (electric) attraction, and short range (Pauli exclusion principle) repulsion between atoms in gas and liquids (Hirschfelder et al. 1966). In summary, these simple considerations show that by immersing two

\footnotetext{
${ }^{1}$ Conservativity is here trivially ensured by the integrability of $1 / z^{2}$.
} 
charged particles rigidly attached in a one-dimensional electric field, one can emulate a restoring force $F_{r}(X)=F(X)-F_{d}$ around any desired position $X_{d}$. Moreover, by tuning the electric charges according to $(7)$, one can remove $F_{d}$ from the model and ensure the passivity of the resulting model. In the rest of the article, these considerations will be extended (from $\mathcal{C}=\mathbb{R}$ to $\mathcal{C}=S E(2))$ to build the wrench of emulated restoring forces acting on our electric probe.

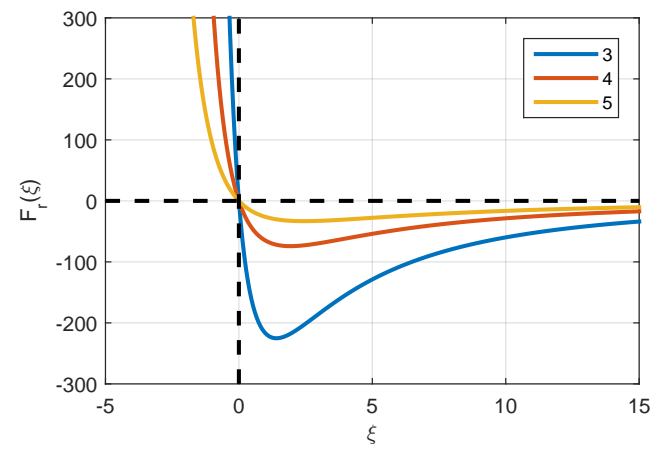

(a)

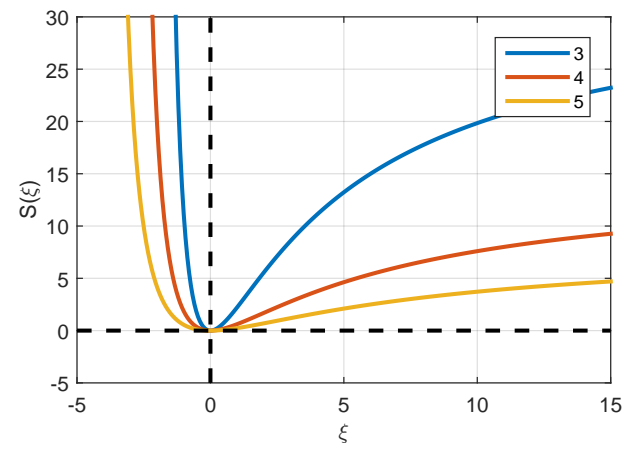

(b)

Figure 5: a: Restoring force (9). b: Storage function (11) for $q=1, l=0.03$ and $\eta_{d}=3,4,5$.

\section{Model of the environment}

In this section, we deal with the "Environment"-block of figure 2. This model is subdivided into several sub-models. One is a geometric model of the scene. The others are related to the electric field and its measurement that will be exploited to build the forces fed back to the operator.

\subsection{Geometric model of the scene}

From now on, the index $d$ will indicate that a configuration dependent function is evaluated on the desired pose of the probe, while for any tensor $T,{ }^{i} T$ will denote the matrix of its components in a frame $\mathcal{F}_{i}$, all the frames being oriented directly. As discussed in the introduction, for the purpose of theoretical analysis, we consider a scene consisting of an infinite vertical wall and an active electric probe able to move in a plane perpendicular to the wall. The wall is assumed to be insulating and using the method of images (Jackson 1999), its electric influence is equivalent to that of the mirror image of the probe reflected by the wall. As a result, the scene can be alternatively defined as a set of two electric active objects: the real probe and its mirror image (see figure 6). These two objects move in the horizontal plane endowed with a fixed inertial frame $\mathcal{F}_{s}=\left(O_{s}, e_{x}, e_{y}\right)$. The probe is a rigid body equipped with a body-attached frame $\mathcal{F}_{p}=\left(O_{p}, e_{\|}, e_{\perp}\right)$ moving in translation and yaw rotation. The image reflected by the wall is itself endowed with a body-attached frame $\mathcal{F}_{w}=\left(O_{w}, e_{w, x}, e_{w, y}\right)$ as indicated in figure 6-a. Any of these frames, say $\mathcal{F}_{i}, i \in\{w, p, s\}$, is parameterized with respect to any other one, say $\mathcal{F}_{j}, j \in\{w, p, s\}$, with a planar rigid transformation (translation and rotation) of $\mathbb{R}^{2}$, i.e., with an element ${ }^{j} g_{i}$ of the Lie group $S E(2)$. In particular, the configurations of the probe with respect to $\mathcal{F}_{s}=\left(O_{s}, e_{s, x}, e_{s, y}\right)$ are defined by the matrices of $S E(2)$

$$
{ }^{s} g_{p}=\left(\begin{array}{cc}
R(\theta) & r \\
0 & 1
\end{array}\right)
$$


where ${ }^{s} R_{p}=R(\theta)$ is the planar rotation matrix mapping $\left(e_{s, x}, e_{s, y}\right)$ onto $\left(e_{\|}, e_{\perp}\right)$, and ${ }^{s} O_{p}=r=$ $(x, y)^{T}$ is the vector of the positional coordinates of $O_{p}$ in $\mathcal{F}_{s}$ as this is depicted in figure 6-a. The transformations (12) are parameterized by the vector $X_{s}=(x, y, \theta)^{T} \in \mathbb{R}^{3}$. As regards the transformation ${ }^{s} g_{w}$, due to the symmetry of the scene, it is given by:

$$
{ }^{w} g_{p}=\left(\begin{array}{cc}
R(2 \alpha) & 2 \rho(\cos \alpha, \sin \alpha)^{T} \\
0 & 1
\end{array}\right),
$$

and is parameterized by $(\rho, \alpha)$ only, where $\rho$ denotes the distance from $O_{p}$ to the wall and $\alpha$ is the oriented angle between $\overrightarrow{O_{w} O_{p}}$ and $\vec{e}_{\|}$, with $O_{w}$ the center of the image (see figure 6 -a).

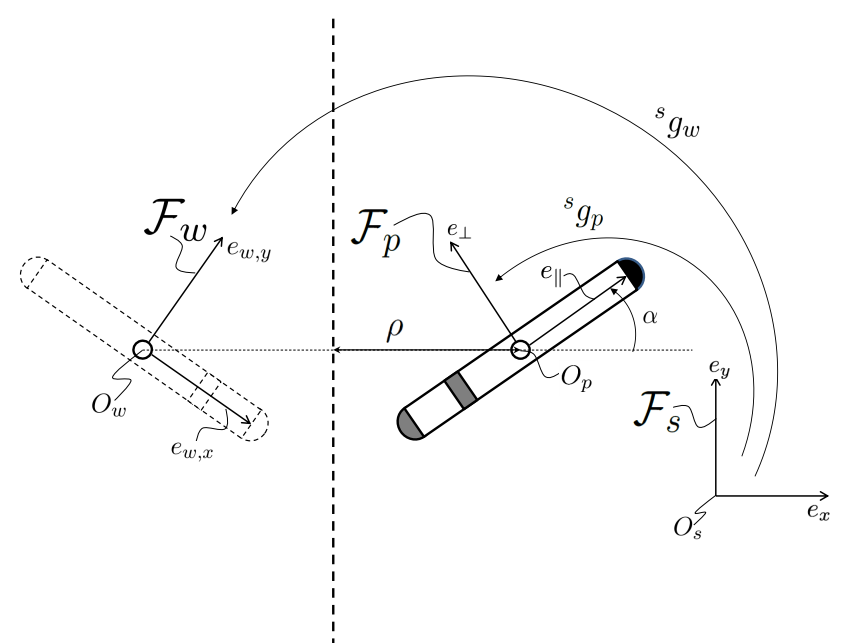

(a)

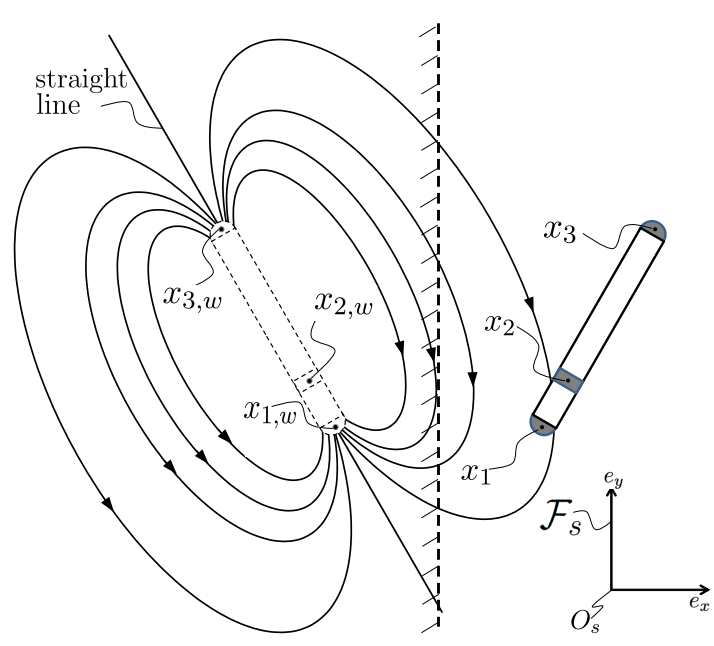

(b)

Figure 6: (a) Parametrization of the scene in the slave space. (b) Portrait of the electric field generated by the mirror image of the probe in the wall.

Note that in all the subsequent developments, the pose ${ }^{s} g_{p}$ is known by feeding a geometric model of the slave robot (our gantry), with its joint variables measurements. On the other hand, the wall position being unknown in $\mathcal{F}_{s}$, the pose of the mirror image ${ }^{s} g_{w}$ is a priori unknown.

\subsection{Model of the reflected electric field}

As evoked in the previous section, the electric influence of the wall is equivalent to that of the mirror image of the probe. Applying laws of electrokinetics (Jackson 1999) to the 3-electrode probe presented in section 2 , its mirror image generates an electric potential of the form:

$$
{ }^{s} x \in \mathbb{R}^{2} \mapsto \phi\left({ }^{s} x\right)=\frac{1}{4 \pi \gamma_{0}} \sum_{i=1}^{3} \frac{I_{i}^{(0)}}{r_{i, w}} \in \mathbb{R},
$$

where $i=1,2,3$ denotes the index of an electrode, ${ }^{s} x_{i, w}$ is the position of its mirror image in $\mathcal{F}_{s}$, while $r_{i, w}=\left\|{ }^{s} x-{ }^{s} x_{i, w}\right\|$ stands for the distance between this mirror electrode and the point ${ }^{s} x$ in which the field is evaluated. Regarding electric quantities, $I_{i}^{(0)}$ is the electric current flowing out 
of the electrode $i$ in an homogeneous unbounded medium of conductivity $\gamma_{0}$ (Boyer et al. 2012). Taking the gradient of (14), provides the model of the reflected electric field:

$$
{ }^{s} x \in \mathbb{R}^{2} \mapsto{ }^{s} E\left({ }^{s} x\right)=-{ }^{s}\left(\nabla_{s_{x}} \phi\left({ }^{s} x\right)\right)=\frac{1}{4 \pi \gamma_{0}} \sum_{i=1}^{3} \frac{I_{i}^{(0)}\left({ }^{s} x-{ }^{s} x_{i, w}\right)}{\left(r_{i, w}\right)^{3}} \in \mathbb{R}^{2} .
$$

\subsection{Model of the electric measurements}

Referring to section 2.1, our probe is constituted of a plastic insulating tube on which are aligned 3 electrodes noted $\varepsilon_{k}$, where $k=1,2,3$, numbers the head, neck and tail groups respectively (see figure $7(\mathrm{a})$ ). The tail electrode is the emitter. The head and neck groups $\varepsilon_{1}$ and $\varepsilon_{2}$ are the receivers, which are subdivided into two symmetrical left and right electrodes. The electric current flowing across each of these sub-electrodes is measured by an electronic board (Servagent et al. 2013). In section 7 we will build a model of the electric forces exerted by the wall onto the probe. To this end, we need first a model of the measured currents when the probe is immersed in the reflected electric field (15) with potential (14). Referring to (Boyer et al. 2012), such a model is naturally expressed in terms of the following combinations :

$$
k=1,2: \delta I_{\mathrm{axk}}=\frac{I_{k}^{\mathrm{1}}+I_{k}^{\mathrm{r}}}{2}, \delta I_{\mathrm{lat} k}=\frac{I_{k}^{1}-I_{k}^{\mathrm{r}}}{2},
$$

where the upper indices $\mathrm{r}$ and $\mathrm{l}$ indicate whether the current is measured by the left or the right sub-electrode of $\varepsilon_{k}$. In (16), $\delta I_{\text {axk }}$ denote the axial component of currents flowing into $\varepsilon_{k}$. As shown in (Boyer et al. 2012), the vector of axial currents $\delta_{\mathrm{ax}}=\left(\delta_{\mathrm{ax} 1}, \delta_{\mathrm{ax} 2}\right)^{T}$ models the response of the probe when it is set under voltage along its axis according to the matrix relation:

$$
\delta I_{\mathrm{ax}}=-C^{(0)} \Phi,
$$

where $C^{(0)}$ is the $3 \times 3$ symmetric, but not invertible ${ }^{2}$, conductivity matrix of the probe with no object in the scene. The minus sign in (17) means that the probe opposes its response to the imposed external potentials $\Phi=\left(\Phi_{1}, \Phi_{2}, \Phi_{3}\right)^{T}$, with $\Phi_{k}=\phi\left({ }^{s} x_{k}\right), \phi$ defined by (14), and ${ }^{s} x_{k}$, the position of $\varepsilon_{k}$ in $\mathcal{F}_{s}$. For the purpose of illustration, using a Boundary Elements Method (BEM) which numerically solves the Laplace equations for any geometry (Boyer et al. 2012), the $C^{(0)}$ matrix of the 3-electrode sensor pictured in figure 1-b, takes the value in Siemens (S):

$$
C^{(0)}=\frac{\gamma_{0}}{100}\left(\begin{array}{ccc}
6.00 & -3.25 & -3.00 \\
-3.25 & 6.50 & -3.50 \\
-3.00 & -3.50 & 6.50
\end{array}\right) \text {. }
$$

Similarly, (16) defines the vector of lateral currents $\delta I_{\text {lat }}=\left(\delta I_{\text {lat1 }}, \delta I_{\text {lat2 }}\right)^{T}$, which models the local response of the sensor to the lateral (perpendicular to the probe axis) component of the external electric vector field as:

$$
k=1,2: \delta I_{\text {latk }}=p_{\perp k} E_{k \perp},
$$

where $E_{k \perp}={ }^{s} e_{\perp}^{T s} E\left({ }^{s} x_{k}\right)$ is the lateral component of the electric field (15) at the center of $\varepsilon_{k}$ of position ${ }^{s} x_{k}$, while the $p_{\perp k}$ 's are some positive scalars standing for the "lateral polarization coefficients" of the probe (Boyer et al. 2012). For instance, for the 3-electrode probe of figure 1-b, a BEM calculation gives $p_{\perp 1}=0.189 \times 10^{-4}$ and $p_{\perp 2}=1.148 \times 10^{-4}$, both expressed in $\Omega \mathrm{m}^{-1}$. Finally, the vectors of lateral and axial currents $\delta I_{\mathrm{ax}}$ and $\delta I_{\text {lat }}$, are with ${ }^{s} g_{p}$, all the information that will be required by our model of emulated electric forces.

\footnotetext{
${ }^{2}$ The currents are in fact not independent since they have to satisfy the conservation of electric charges.
} 


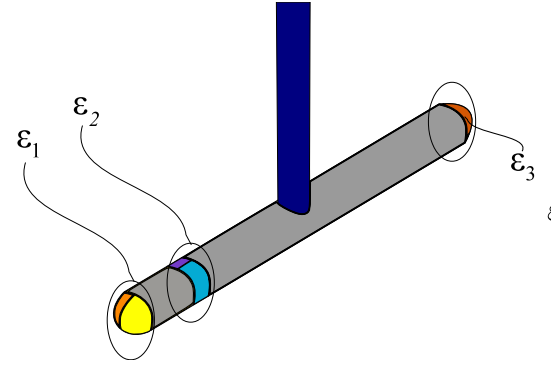

(a)

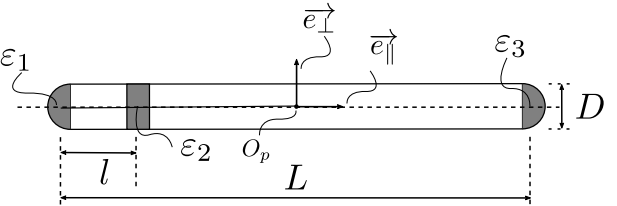

(b)

Figure 7: (a) Schematize of a 3-electrode probes. (b) Parametrization of the probe in its frame.

\subsection{Model of the electric field along the probe}

In section 7 , the forces exerted by the wall onto the probe will be emulated by a set of virtual Coulomb charges located on $\epsilon_{k}, k=1,2$. In this perspective, we now derive a model able to estimate the electric field at the electrodes center, from the knowledge of the measured axial and lateral currents (16). Regarding the lateral components $E_{\perp}={ }^{s} e_{\perp}^{T s} E$, the expected model is directly deduced by inverting the local relations (19) which gives,:

$$
k=1,2: E_{k \perp}=A_{\perp k} \delta I_{\text {latk }},
$$

where we introduced the notations $A_{\perp k}=p_{\perp k}^{-1}$. To derive a similar model of the parallel components of the field $E_{\|}={ }^{s} e_{\|}^{T s} E$, we first define the electric potential along the probe as the function:

$$
\phi_{\|}(r, \theta, \ell)=\phi\left({ }^{s} x_{3}+\ell{ }^{s} e_{\|}\right),
$$

with ${ }^{s} x_{3}=r+(L / 2)(\cos (\theta), \sin (\theta))^{T}$, and $\ell \in \mathbb{R}$ is a (signed) abscissa running along the probe axis and counted from $\varepsilon_{3}$. Then, we approximate $\phi_{\|}$with a second order polynomial interpolation:

$$
\tilde{\phi}_{\|}(r, \theta, \ell)=a_{0}+a_{1} \ell+a_{2} \ell^{2}
$$

where $a_{i=0,1,2}$ are some pose-dependent coefficients, solutions of the system:

$$
\left(\begin{array}{ccc}
1 & \ell_{1} & \ell_{1}^{2} \\
1 & \ell_{2} & \ell_{2}^{2} \\
1 & \ell_{3} & \ell_{3}^{2}
\end{array}\right)\left(\begin{array}{l}
a_{0} \\
a_{1} \\
a_{2}
\end{array}\right)=\left(\begin{array}{c}
\Phi_{1} \\
\Phi_{2} \\
\Phi_{3}
\end{array}\right)
$$

and $\ell_{i}, i=1,2,3$ are the axial abscissae of $\epsilon_{i=1,2,3}$ counted from $\epsilon_{3}$, i.e., $\ell_{1}=-L, \ell_{2}=-(L-l)$, $\ell_{3}=0$. Now, referring to (Boyer et al. 2012), one can consider the reduced system extracted from (17):

$$
\left(\begin{array}{l}
\delta I_{\mathrm{ax} 1} \\
\delta I_{\mathrm{ax} 2}
\end{array}\right)=\left(\begin{array}{ll}
C_{11}^{(0)} & C_{12}^{(0)} \\
C_{21}^{(0)} & C_{22}^{(0)}
\end{array}\right)\left(\begin{array}{c}
\Phi_{1}-\Phi_{3} \\
\Phi_{2}-\Phi_{3}
\end{array}\right) .
$$

In contrast to the full $3 \times 3$ matrix $C^{(0)}$ of (17), the above $2 \times 2$ matrix is not only symmetric but also invertible, and because $\ell_{3}=0 \Rightarrow a_{0}=0$, and one can write

$$
\left(\begin{array}{l}
a_{1} \\
a_{2}
\end{array}\right)=\left(\begin{array}{ll}
\ell_{1} & \ell_{1}^{2} \\
\ell_{2} & \ell_{2}^{2}
\end{array}\right)^{-1}\left(\begin{array}{ll}
C_{11}^{(0)} & C_{12}^{(0)} \\
C_{21}^{(0)} & C_{22}^{(0)}
\end{array}\right)^{-1}\left(\begin{array}{l}
\delta I_{\mathrm{ax} 1} \\
\delta I_{\mathrm{ax} 2}
\end{array}\right) .
$$


From this result, one can deduce the following approximation of the axial electric field along the probe

$$
\tilde{E}_{\|}(\ell)=-\frac{\partial \tilde{\phi}_{\|}}{\partial \ell}(r, \theta, \ell)=-a_{1}-2 a_{2} \ell=-(1,2 \ell)\left(\begin{array}{c}
a_{1} \\
a_{2}
\end{array}\right)
$$

which provides the approximation of its values $E_{\|}\left(\ell_{k}\right)=E_{k \|}$ on the electrodes $\varepsilon_{k}$ :

$$
k=1,2: E_{k \|} \simeq A_{\| k 1} \delta I_{\mathrm{ax} 1}+A_{\| k 2} \delta I_{\mathrm{ax} 2}
$$

where using (25) and (26), one has:

$$
\left(\begin{array}{cc}
A_{\| 11} & A_{\| 12} \\
A_{\| 21} & A_{\| 22}
\end{array}\right)=-\left(\begin{array}{cc}
1 & 2 \ell_{1} \\
1 & 2 \ell_{2}
\end{array}\right)\left(\begin{array}{cc}
\ell_{1} & \ell_{1}^{2} \\
\ell_{2} & \ell_{2}^{2}
\end{array}\right)^{-1}\left(\begin{array}{cc}
C_{11}^{(0)} & C_{12}^{(0)} \\
C_{21}^{(0)} & C_{22}^{(0)}
\end{array}\right)^{-1}
$$

Note that (27) is merely the axial counterpart of (20). Finally, the coefficients $A_{\perp k}$ and $A_{\| k l}$ define a set of constant parameters (having for dimension $\Omega^{-1} \mathrm{~m}$ ) that only depend on the sensor design, and not on the pose of the probe.

\subsection{Assessment of the model of the electric field along the probe}

We now consider the 3-electrode probe pictured in figure 1-b. Its geometric parameters are $l=$ $0.04 \mathrm{~m}, L=0.21 \mathrm{~m}, D=0.02 \mathrm{~m}$. With such a design, using (18) and the values of the lateral polarization coefficients after (19), the $A_{\perp}$ and $A_{\|}$-matrices of the above analytical model are (in $\left.\Omega \mathrm{m}^{-1}\right)$ :

$$
\left(\begin{array}{cc}
A_{\perp 1} & 0 \\
0 & A_{\perp 2}
\end{array}\right)=\left(\begin{array}{cc}
5.29 & 0 \\
0 & 6.75
\end{array}\right) 10^{4}, \quad\left(\begin{array}{ll}
A_{\| 11} & A_{\| 12} \\
A_{\| 21} & A_{\| 22}
\end{array}\right)=\left(\begin{array}{ll}
5.1497 & -5.8237 \\
3.6415 & -1.6150
\end{array}\right) 10^{3} .
$$

In order to assess the analytical model of the field in section 6.4, we compare its predictions for our 3-electrode probe to those predicted by the BEM. Assuming that $\mathcal{F}_{s}$ is aligned with the wall according to figure 7 , the probe is first positioned in $X_{0}=\left(x_{0}, 0,0\right)^{T}$ with $x_{0}$ such that the distance between $O_{p}$ and the wall is $15 \mathrm{~cm}$. The probe is then moved along:

- A $x$-path such that $(y, \theta)=(0,0)$, and $x$ runs between $0 \mathrm{~m}$ and $0.3 \mathrm{~m}$,

- a $y$-path such that $(x, \theta)=\left(x_{0}, 0\right)$, and $y$ runs between $-0.4 \mathrm{~m}$ and $0.4 \mathrm{~m}$,

- a $\theta$-path such that $(x, y)=\left(x_{0}, 0\right)$, and $\theta$ runs between $-\pi / 2$ and $\pi / 2$,

The axial and lateral components $E_{k \|}$ and $E_{k \perp}$ are then calculated with the BEM and the analytical approximations $(20,27,29)$ fed by the electric currents calculated by the BEM. The results of these calculations are illustrated in Figures 8 and 9 which display the non-zero plots provided by these two models for the $x, y$, and $\theta$-path. In all these simulations, the analytical approximation of the electric field based on the measurements fits well with the BEM. In particular, both models inherit the basic properties of the reflected field. Along $\theta$-paths, $E_{k \|}$ and $E_{k \perp}$ are symmetric and skew-symmetric respectively, with respect to (w.r.t.) $\left(x_{0}, 0,0\right)$. Along the $y$-path, $E_{k \|}$ remains constant while $E_{k \perp}=0$. Finally, as expected from laws of electrostatics, along the $x$-path, $E_{k \|}$ drops as the inverse of the square of the distance between the probe and the wall. 


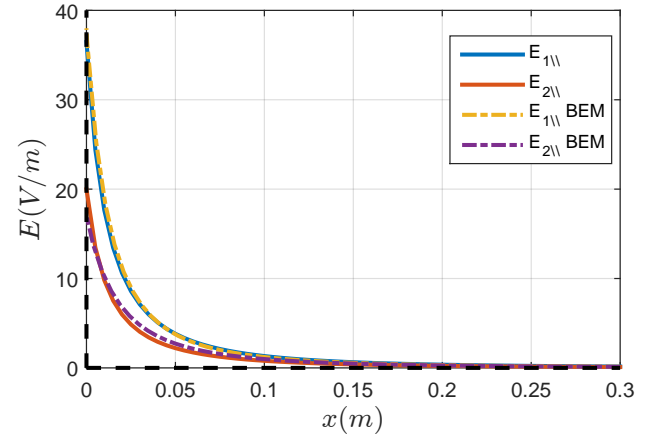

(a)

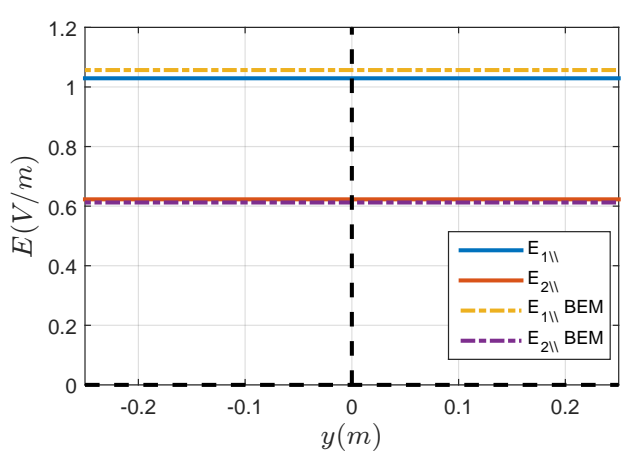

(b)

Figure 8: Axial components of the electric field at each measurement electrodes along the $x$ (left) and $y$-path (right): comparison between BEM (dashed lines) and analytical model (solid lines).

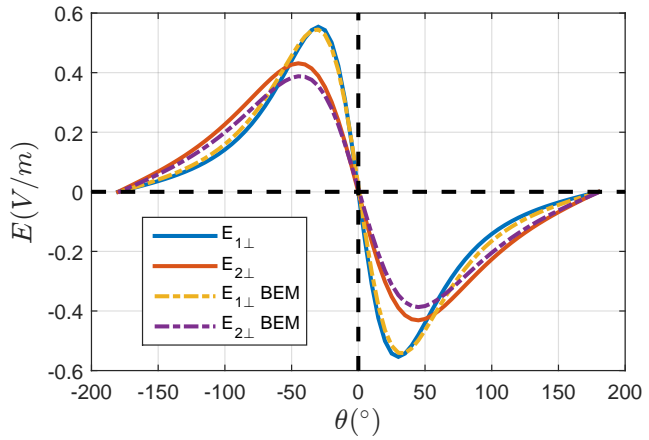

(a)

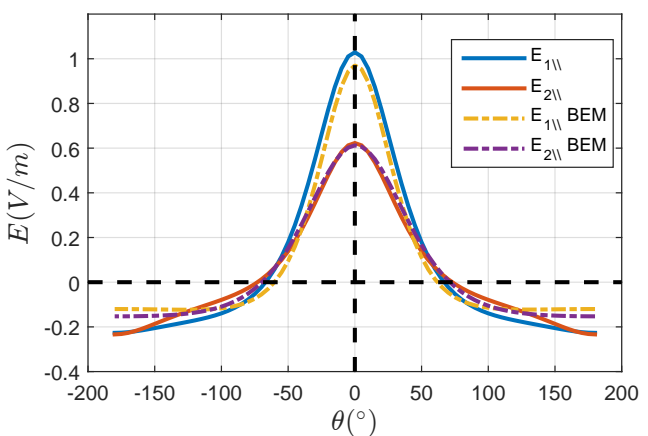

(b)

Figure 9: Lateral and axial components of the electric field along the $\theta$-path: comparison between BEM (dashed lines) and analytical model (solid lines).

\section{Emulation of the electric restoring forces}

In this section we use the above measurements with the aim of building a model of the pre-touch forces exerted by the wall onto the probe. These forces will be fed-back to the operator through the haptic interface as presented in section 2. As mentioned in introduction, this model consists of some restoring forces that tend to steer the probe in a pose perpendicular to the wall. In such a desired pose, the head of the probe faces the wall at a prescribed distance $d-L / 2$ from it. Due to the symmetry of the scene, it exists an infinity of such desired poses ${ }^{s} g_{p_{d}}$. However, once expressed in the frame of the mirror image $\mathcal{F}_{w}$, the desired pose ${ }^{w} g_{p_{d}}$ is unique. In words, ${ }^{w} g_{p_{d}}$ given by (13) with $(\rho, \alpha)=(d, 0)$, defines the desired pose relative to the wall regardless of its particular position along it. This context is illustrated by the figure 1 with the parameters of figure 6 -a. With this picture in mind, we will build upon the basic concepts of sections 4 and 5, by imposing to our model of restoring forces, the further requirements:

- Requirement 1: The model needs to be conservative.

- Requirement 2: It's a function of measurements $\delta I_{\mathrm{ax}}, \delta I_{\text {lat }}$ and the probe pose ${ }^{s} g_{p}$ only. 
- Requirement 3: The desired pose ${ }^{w} g_{p_{d}}$ is an equilibrium pose of the model.

- Requirement 4: The desired pose is the unique minimum of the associated $S$ function.

Note that requirements 1,3 and 4 are nothing else than the conditions required by our model of passive restoring force as defined at the end of section 4 , with $S$ the corresponding storing function. As regards requirement 2, let us also remind that while the poses ${ }^{s} g_{p}$ and ${ }^{w} g_{p_{d}}$ are known (the first is deduced with the geometric model of the slave, the second is prescribed), the pose ${ }^{w} g_{p}$ is of course a priori unknown, and should be steered toward ${ }^{w} g_{p_{d}}$ by our emulated restoring forces. In the subsequent developments we will address these requirements step by step. To that end, we will build on the model of Coulomb forces of electrostatics as it is reminded in the next subsection.

\subsection{Reminder of electrostatics}

In the subsequent developments we will base our emulation of restoring forces on basic facts of electrostatics (Jackson 1999) in continuity with the Lennard-Johns model of section 5. First, let us consider an electric field $x \in \mathbb{R}^{3} \mapsto E(x) \in \mathbb{R}^{3}$ (for the sake of concision, the index of the expression frame is omitted). Then, any electric charge $q \in \mathbb{R}$ of position vector $x$ in space, is subject to the Coulomb force:

$$
F(x)=q E(x) .
$$

In the same way, if the field $E$ varies enough slowly with respect to $x$, then any electric dipole $p \in \mathbb{R}^{3}$ immersed in $E$, is subject to no force but to the Coulomb couple:

$$
C(x)=p \times E(x),
$$

which tends to align the dipole with the field. Moreover, Coulomb forces and couples are conservative.

\subsection{Requirement 1: Emulation of virtual Coulomb charges and dipoles}

To properly emulate a system of external conservative forces exerted onto the probe by the environment (its mirror image reflected by the wall), one can use the above model of electrostatic forces and torques. The key idea thus consists of using the measurements of the probe to emulate a set of virtual Coulomb charges and dipoles rigidly attached to the probe considered as a compass that can move and rotate freely under the influence of the reflected electric field. These virtual Coulomb charges and dipoles are positioned at the centers of the measurement electrode groups $\varepsilon_{k}, k=1,2$. Thus, any $\varepsilon_{k}$ is paired with a virtual electric charge $q_{k}$ and a virtual electric dipole ${ }^{p} p_{k} \in \mathbb{R}^{2}$. Regarding dipoles, they are imposed to be aligned with the probe axis, i.e., ${ }^{p} p_{k}=\left(p_{k}, 0\right)^{T}$ in order to generate an alignment torque with the local value of the measured electric field. Hence, referring to section 7.1, we can define for any $\varepsilon_{k}$ the following external force and torque expressed in the probe frame

$$
{ }^{p} F_{k}=q_{k}^{p} E_{k}, C_{k}=p_{k} E_{k \perp},
$$

where we used the fact that a virtual dipole is aligned with the probe, while for the sake of concision, we shall note from now on ${ }^{p} E\left({ }^{s} x_{k}\right)={ }^{p} E_{k}=\left(E_{k \|}, E_{k \perp}\right)^{T}$. In the case of our 3-electrode probe, because the electric field can be measured on $\varepsilon_{1}$ and $\varepsilon_{2}$ only, the superimposition principle allows writing the wrench of virtual electric forces in the general form:

$$
{ }^{p} \mathbb{F}=\left(\begin{array}{c}
{ }^{p} F \\
M
\end{array}\right)=\left(\begin{array}{c}
{ }^{p} F_{1}+{ }^{p} F_{2} \\
C_{1}+C_{2}-l_{1} F_{\perp 1}-l_{2} F_{\perp 2}
\end{array}\right),
$$




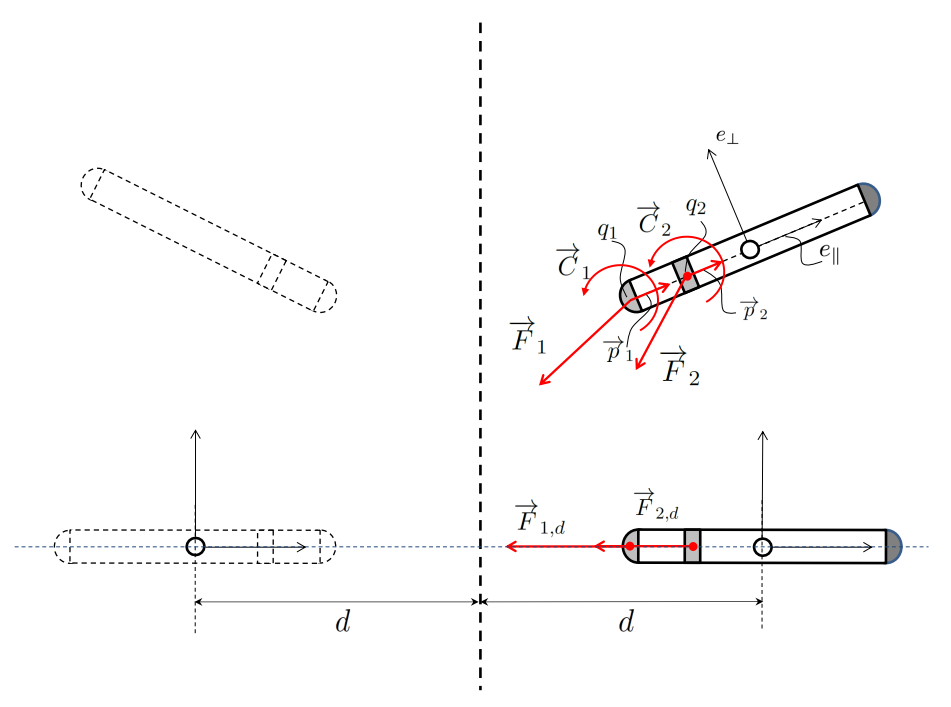

Figure 10: Model of virtual electric charges and dipoles.

where $l_{1}=L / 2$ and $l_{2}=(L / 2)-l$ are the distances between $\varepsilon_{1}$ and $O_{p}$, and $\varepsilon_{2}$ and $O_{p}$, respectively (see figure 7-b). Such a configuration of charges and dipoles is illustrated in figure 10. According to section 7.1 and superposition principle, the wrench (33) is conservative and does fulfill requirement 1. Starting from this general configuration of charges and dipoles, we will impose to it some restrictions in order to fulfill the three further requirements.

\subsection{Requirement 2: Sensor-based model of electric forces}

We now address the second requirement of the beginning of section 7, i.e., we check whether the virtual forces and torques of (33) can be built from the knowledge of our embarked measurements and the pose ${ }^{s} g_{p}$, only.

\subsubsection{Sensor based model of Coulomb forces and couples}

Let us express the virtual Coulomb forces and torques induced by a charge and a dipole $\left(q_{k}, p_{k}\right)$ placed in $\varepsilon_{k}$ in terms of the reflected electric field. In a frame $\mathcal{F}_{p_{\varepsilon_{k}}}$ centered on $\varepsilon_{k}$ with vector basis $\left(e_{\|}, e_{\perp}\right)$, we have from (32):

$$
p_{\varepsilon_{k}} \mathbb{F}_{k}=\left(\begin{array}{c}
q_{k} E_{k \|} \\
q_{k} E_{k \perp} \\
p_{k} E_{k \perp}
\end{array}\right) .
$$

Hence, if for any group $\varepsilon_{k}$, one can derive an expression of $E_{k \|}$ and $E_{k \perp}$ in terms of the measured currents $\delta I_{\text {ax }}$ and $\delta I_{\text {lat }}$ only, the model fulfills our requirement 2. Indeed, referring to section 6.4, $E_{k \perp}$ and $E_{k \|}$ can be imaged by $\delta I_{\text {lat }}$ and $\delta I_{\text {ax }}$ as follow:

$$
{ }^{p} E_{k}=\left(\begin{array}{c}
E_{k \|} \\
E_{k \perp}
\end{array}\right)=\left(\begin{array}{c}
A_{\| k 1} \delta I_{\mathrm{ax} 1}+A_{\| k 2} \delta I_{\mathrm{ax} 2} \\
A_{\perp k} \delta I_{\mathrm{lat} k}
\end{array}\right) .
$$


Finally, inserting (35) into (34) shows that a Coulomb charge and dipole located in $\varepsilon_{k}$ can be emulated by the measure-based model:

$$
p_{\varepsilon_{k}} \mathbb{F}_{k}=\left(\begin{array}{c}
{ }^{p} F_{k} \\
C_{k}
\end{array}\right)=\left(\begin{array}{c}
F_{k \|} \\
F_{k \perp} \\
C_{k}
\end{array}\right)=\left(\begin{array}{c}
K_{\| k 1} \delta I_{\mathrm{ax} 1}+K_{\| k 2} \delta I_{\mathrm{ax} 2} \\
K_{\perp k} \delta I_{\mathrm{lat} k} \\
K_{\theta k} \delta I_{\mathrm{lat} k}
\end{array}\right)
$$

where $K_{\| k l}, K_{\perp k}$ and $K_{\theta k}$ are four design constants (four gains) parameterized by the virtual charges $q_{k}$ and the virtual dipoles $p_{k}$ through the relations:

$$
k=1,2: K_{\| k l}=q_{k} A_{\| k l}, K_{\perp k}=q_{k} A_{\perp k}, K_{\theta k}=p_{k} A_{\perp k} .
$$

These four gains being pose-independent, each ${ }^{p_{\varepsilon_{k}}} \mathbb{F}_{k}$ depends on the measurements only, and thus satisfies the requirement 2. Now, let us apply these results to our configuration of charges, i.e., one charge and one dipole on the neck and the head electrode. In this case, the wrench of emulated electric forces (33) reads

$$
{ }^{p} \mathbb{F}=\left(\begin{array}{c}
{ }^{p} F \\
M
\end{array}\right)=\left(\begin{array}{c}
{ }^{p} F_{1}+{ }^{p} F_{2} \\
C_{1}+C_{2}-l_{1} F_{1, \perp}-l_{2} F_{2, \perp}
\end{array}\right)=\left(\begin{array}{c}
q_{1} E_{1 \|}+q_{2} E_{2 \|} \\
q_{1} E_{1 \perp}+q_{2} E_{2 \perp} \\
\left(p_{1}-l_{1} q_{1}\right) E_{1 \perp}+\left(p_{2}-l_{2} q_{2}\right) E_{2 \perp}
\end{array}\right),
$$

which can be detailed in terms of measurements as

$$
p_{\mathbb{F}}=\left(\begin{array}{c}
K_{\| 1}^{*} \delta I_{\mathrm{ax} 1}+K_{\| 2}^{*} \delta I_{\mathrm{ax} 2} \\
K_{\perp 1} \delta I_{\mathrm{lat} 1}+K_{\perp 2} \delta I_{\mathrm{lat} 2} \\
K_{\theta 1}^{*} \delta I_{\mathrm{lat} 1}+K_{\theta 2}^{*} \delta I_{\mathrm{lat} 2}
\end{array}\right)
$$

with the further notations:

$$
K_{\| 1}^{*}=K_{\| 11}+K_{\| 21}, K_{\| 2}^{*}=K_{\| 12}+K_{\| 22}, K_{\theta 1}^{*}=K_{\theta 1}-l_{1} K_{\perp 1}, K_{\theta 2}^{*}=K_{\theta 2}-l_{2} K_{\perp 2},
$$

which will be systematically used in the subsequent developments. Note that in these expressions, the star indicates that the definition of the gain involves non-local measurements (introduced by the momentums of forces or by the coupled model of $\left.E_{\|}\right)$.

\subsubsection{Sensor-based model of the restoring wrench}

To achieve the design of our emulated wrench, we need to ensure it to represent a system of restoring forces. To that end, we prolong the idea developed in the simple illustrative one-dimensional example of section 5. We first define the desired wrench as that exerted by the virtual Coulomb forces and torques when the probe is in its desired pose with respect to its mirror image. Using (39), such a wrench is defined by its components in the (desired) probe frame:

$$
p_{d} \mathbb{F}_{d}=\left(\begin{array}{c}
{ }^{p_{d}} F_{d} \\
M_{d}
\end{array}\right)=\left(\begin{array}{c}
q_{1}{ }^{p_{d}} E_{1 d}+q_{2}{ }^{p_{d}} E_{2 d} \\
0
\end{array}\right)=\left(\begin{array}{c}
K_{\| 1}^{*} \delta I_{\mathrm{ax} 1 d}+K_{\| 2}^{*} \delta I_{\mathrm{ax} 2 d} \\
0 \\
0
\end{array}\right),
$$

where we used the fact that for any pose aligned with the mirror image, the symmetry of the scene imposes $\delta I_{\text {lat } i}=p_{\perp i} E_{i \perp}=0, i=1,2$. 
Then, the emulated wrench is obtained by subtracting this desired wrench from the corresponding electric wrench in the current pose. Using the definition of the probe configuration space as the Lie group $S E(2)$, when comparing ${ }^{p} \mathbb{F}$ and ${ }^{p_{d}} \mathbb{F}_{d}$ through this substraction, we should use the transport of wrenches:

$$
{ }^{p} \mathbb{F}_{r}={ }^{p} \mathbb{F}-A d_{p_{d} g_{p}}^{T} p_{d} \mathbb{F}_{d},
$$

where $A d_{p_{d} g_{p}}$ is the adjoint action of $S E(2)$ onto its Lie algebra se(2) (Murray et al. 1994), which depends on the probe pose ${ }^{p_{d}} g_{p}$ and thus does not satisfy our requirement 2 . Therefore, to preserve this requirement, we use the idea presented in section 5 and fix the particles charge such that ${ }^{p_{d}} F_{d}=0$ (remind that the alignment already imposes $M_{d}=0$ ), i.e., we impose the condition on the two charges:

$$
q_{1}{ }^{p_{d}} E_{1 d}+q_{2}{ }^{p_{d}} E_{2 d}=0 \Rightarrow \frac{q_{2}}{q_{1}}=-\frac{E_{1 \| d}}{E_{2 \| d}} \triangleq-\alpha_{E d}
$$

with $q_{1}>0$ and where we used the fact that due to symmetry, $E_{1 \perp d}=E_{2 \perp d}=0$. In short, imposing (43), the desired force disappears from the model (42) which then enjoys the requirement 2, while being consistent with the comparison of two wrenches in the same basis of $s e(2)^{\star}$. Finally, in the probe frame, this model of the restoring electric forces simply reads:

$$
{ }^{p} \mathbb{F}_{r}=\left(\begin{array}{c}
{ }^{p} F_{r} \\
M_{r}
\end{array}\right)=\left(\begin{array}{c}
{ }^{p} F-0 \\
M-0
\end{array}\right)=\left(\begin{array}{c}
F_{r \|} \\
F_{r \perp} \\
M_{r}
\end{array}\right)=\left(\begin{array}{c}
q_{1}\left(E_{1 \|}-\alpha_{E d} E_{2 \|}\right) \\
q_{1}\left(E_{1 \perp}-\alpha_{E d} E_{2 \perp}\right) \\
\left(p_{2}+l_{2} \alpha_{E d} q_{1}\right) E_{2 \perp}+\left(p_{1}-l_{1} q_{1}\right) E_{1 \perp}
\end{array}\right) .
$$

So far, the model of electric forces has been expressed in the mobile probe frame. However, for the purpose of position-position remote control, it is required to transport this model in a frame related to the slave space according to:

$$
s^{\star} \mathbb{F}_{r}=A d_{g_{g^{\star}}}^{T}{ }^{p} \mathbb{F}_{r},
$$

with:

$$
{ }^{s^{*}} \mathbb{F}_{r}=\left(\begin{array}{c}
{ }^{s} F_{r} \\
M_{r}
\end{array}\right)=\left(\begin{array}{c}
{ }^{s} R_{p}^{p} F_{r} \\
M_{r}
\end{array}\right)
$$

and ${ }^{p} F_{r}=\left(F_{r \|}, F_{r \perp}\right)^{T}$ defined by (44). In this slave-related model, we used the frame $\mathcal{F}_{s}^{\star}$, indicated by the left upper index " $s^{\star} "$ in (46). This frame is deduced by parallel translating the basis vector of $\mathcal{F}_{s}$ from $O_{s}$ to the probe center $O_{p}$ (see Figure 6). Such a choice is systematically used in the following. It is more intuitive than using $\mathcal{F}_{s}$ since it allows directly feeding back the momentum of Coulomb forces related to the probe center $O_{p}$, to the yaw DoF of the joystick. Finally, the ego-centered model (44) depends only on the electric measurements, while the allo-centered one (45) also depends on the pose ${ }^{s} g_{p}$. Therefore, they both satisfy the requirement 2 .

\subsection{Requirement 3: Equilibrium analysis of the restoring forces}

In this section we study the equilibrium poses of our model of emulated forces, i.e., we seek the poses ${ }^{w} g_{p}$ that ensure ${ }^{p} F_{r}=0$ and $M_{r}=0$. Using (44), these two vector conditions impose the three scalar constraints on the electric field in $\mathcal{F}_{p}$

$$
\begin{aligned}
& F_{r \|}=q_{1}\left(E_{1 \|}-\alpha_{E d} E_{2 \|}\right)=0, \\
& F_{r \perp}=q_{1}\left(E_{1 \perp}-\alpha_{E d} E_{2 \perp}\right)=0, \\
& M_{r}=\left(p_{2}+l_{2} \alpha_{E d} q_{1}\right) E_{2 \perp}+\left(p_{1}-l_{1} q_{1}\right) E_{1 \perp}=0 .
\end{aligned}
$$


According to our requirement 3, this set of 3 algebraic nonlinear equations needs to have as root, the desired pose whose parameters related to the wall are $\left(\rho_{d}, \alpha_{d}\right)=(d, 0)$, with $d$ fixing the distance between the probe and the mirror image when they are aligned as in figure 10.

To achieve this further requirement, it is convenient to impose the following further constraints on our four design parameters $\left(q_{1}, q_{2}, p_{1}, p_{2}\right)$ :

$$
p_{1}=l_{1} q_{1}, p_{2}>l_{2} q_{2}=-l_{2} \alpha_{E d} q_{1} .
$$

This allows changing (47-49) into

$$
F_{r \|}=q_{1}\left(E_{1 \|}-\alpha_{E d} E_{2 \|}\right)=0, F_{r \perp}=q_{1}\left(E_{1 \perp}-\alpha_{E d} E_{2 \perp}\right)=0, M_{r}=\left(p_{2}+l_{2} \alpha_{E d} q_{1}\right) E_{2 \perp}=0 .
$$

To find the roots of (51), one can progress through simple considerations based on the symmetries of the scene and the parameters $(\rho, \alpha)$ of figure 6 . To that end, let us first remark that the two above conditions $F_{r \perp}=0$ and $M_{r}=0$, impose $E_{1, \perp}=E_{2, \perp}=0$, which can hold solely when the probe axis is aligned with the unique straight line of the reflected electric field, i.e., when it is perpendicular to the wall (see figure 6-b). With our parameters, this means that $\alpha=0$ or $\pi$. Now imposing each of these two values in the third condition $F_{r \|}=0$, defines in both cases, a single equation with respect to $\rho$ which reads:

$$
q_{1}\left(E_{1 \|}-\alpha_{E d} E_{2 \|}\right)(\rho)=0 \Leftrightarrow \frac{E_{1 \|}}{E_{2 \|}}(\rho)=\alpha_{E d} .
$$

This equation has no root for $\alpha=\pi$. On the other hand, when $\alpha=0$, according to the definition (43) of $\alpha_{E d}$, (52) has for unique root $\rho=d$ and the requirement 3 is fulfilled. To close this section, one can now state our restoring wrench compatible with requirements 1, 2, 3 in the final form

$$
\left(\begin{array}{c}
F_{r \|} \\
F_{r \perp} \\
M_{r}
\end{array}\right)=\left(\begin{array}{c}
q_{1}\left(E_{1 \|}-\alpha_{E d} E_{2 \|}\right) \\
q_{1}\left(E_{1 \perp}-\alpha_{E d} E_{2 \perp}\right) \\
\left(p_{2}+l_{2} \alpha_{E d} q_{1}\right) E_{2 \perp}
\end{array}\right)
$$

which only depends on the two physical parameters $q_{1}$ and $p_{2}$. Equivalently, using (20), (27) with (37), (40), as well as the fact that the equality constraint of (50) imposes $K_{\theta 1}^{*}=0$, one can obtain the general form of the restoring wrench as a function of the measured currents:

$$
\left(\begin{array}{c}
F_{r \|} \\
F_{r \perp} \\
M_{r}
\end{array}\right)=\left(\begin{array}{c}
K_{\perp 1}\left[\left(\beta_{11}-\beta_{21} \lambda\right) \delta I_{\mathrm{ax} 1}+\left(\beta_{12}-\beta_{22} \lambda\right) \delta I_{\mathrm{ax} 2}\right] \\
K_{\perp 1}\left(\delta I_{\mathrm{lat} 1}-\lambda \delta I_{\mathrm{lat} 2}\right) \\
K_{\theta 2}^{*} \delta I_{\mathrm{lat} 2}
\end{array}\right)
$$

Note that (54) is entirely defined by the two independent gains $K_{\perp 1}=q_{1} A_{\perp 1}$ and $K_{\theta 2}^{*}=\left(p_{2}+\right.$ $\left.l_{2} \alpha_{E d} q_{1}\right) A_{\perp 2}$, as well as the further constants

$$
\beta_{k l}=\frac{A_{\| k l}}{A_{\perp k}}, \lambda=\alpha_{E d} \frac{A_{\perp 2}}{A_{\perp 1}}
$$

which only depend on the probe morphology and the desired pose. 


\subsection{Requirement 4: Passivity of the restoring forces}

In this section we wonder whether the desired pose is the unique minimum of the storing function associated to our virtual wrench ${ }^{s} \mathbb{F}_{r}=\left({ }^{s} F_{r}^{T}, M_{r}\right)^{T}$. To this end, we refer to the general context of section 4 , and remark that for our electric compass, the path integral of (3) is instantiated for any $X_{s}=\left(r^{T}, \theta\right)^{T}$ by:

$$
S\left(X_{s}\right)=-\int_{r_{d}}^{r}{ }^{s} F_{r}^{T} d r-\int_{\theta_{d}}^{\theta} M_{r} d \theta,
$$

in which we have introduced the detailed expression of ${ }^{s} F_{r}$ given by $(46)$ and $\left(d r^{T}, d \theta\right)=(d x, d y, d \theta)$ defines a path in $S E(2)$. Looking at (56), it is straightforward that we have:

$$
{ }^{s} \mathbb{F}_{r}=-\nabla_{X_{s}} S
$$

Thus, as shown in the previous section, $S$ has a unique singularity which is such that the probe is perpendicular to the wall with its head facing it (i.e. on the axis $\alpha=0$ of the $(\rho, \alpha)$ space). In this case we are in the conditions of the Lennard-Johns forces of section 5 and $S \rightarrow+\infty$ when $\rho \rightarrow L / 2$ (i.e. when the head electrode touches its mirror image). Hence, this unique singular point of $S$ can only be a saddle point or a global minimum (not a maximum). But a saddle point would mean that there would exist other singularities of $S$ out of the axis $\alpha=0$, what is impossible. Thus, $S$ has a global minimum for $(\rho, \alpha)=(d, 0)$, and this minimum is such that $S=0$. Therefore, $S$ is nonnegative and does define a storing function. Finally, our model of forces defined by (53) or equivalently (54), does be passive.

\section{Implementation of the model of restoring forces}

The model of Coulomb charges reacting to an electric field, has revealed to be a useful tool for designing $\mathbb{F}_{r}$. However, for its practical implementation, it is preferable to express $\mathbb{F}_{r}$ directly in terms of the measured currents (while preserving the passivity of the model). This practical implementation of $\mathbb{F}_{r}$ is presented in this section. To implement the model of restoring forces, one can first consider it in the form (53), i.e., as a function of the electric field components $E_{k \|}$ and $E_{k \perp}$, which then should be replaced by their expressions in terms of the measured currents as they are given in section 6.4. In particular, the $\alpha_{E d^{-}}$-coefficient can be easily expressed as:

$$
\alpha_{E d}=\frac{A_{\| 11} \delta I_{\mathrm{ax} 1 d}+A_{\| 12} \delta I_{\mathrm{ax} 2 d}}{A_{\| 21} \delta I_{\mathrm{ax} 1 d}+A_{\| 22} \delta I_{\mathrm{ax} 2 d}} .
$$

Adopting this process, one could entirely parameterize the model with the two gains $K_{\perp 1}$ and $K_{\theta_{1}}^{*}$ as this is apparent in the expressions (54), (55). Though this first implementation process is valid, we used an equivalent, but more straightforward alternative based on the general form of the restoring wrench (42), in which one inserts (39) and (41). This gives:

$$
{ }^{p} \mathbb{F}_{r}=\left(\begin{array}{c}
K_{\| 1}^{*} \delta I_{\mathrm{ax} 1}+K_{\| 2}^{*} \delta I_{\mathrm{ax} 2} \\
K_{\perp 1} \delta I_{\mathrm{lat} 1}+K_{\perp 2} \delta I_{\mathrm{lat} 2} \\
K_{\theta 1}^{*} \delta I_{\mathrm{lat} 1}+K_{\theta 2}^{*} \delta I_{\mathrm{lat} 2}
\end{array}\right)-A d_{p_{d} g_{p}}^{T}\left(\begin{array}{c}
K_{\| 1}^{*} \delta I_{\mathrm{ax} 1 d}+K_{\| 2}^{*} \delta I_{\mathrm{ax} 2 d} \\
0 \\
0
\end{array}\right) .
$$

Then, comparing (59) with the general form of the restoring wrench (42), it becomes apparent that one can directly remove the desired component ${ }^{p_{d}} \mathbb{F}_{d}$ in (59) as follows. We first position the 
probe in the desired pose $\left(\rho_{d}, \alpha_{d}\right)=(d, 0)$. Then, we measure the two axial currents of the neck and the head electrodes, and tune the gains $K_{\| 1}^{*}$ and $K_{\| 2}^{*}$ in order to fulfill the condition:

$$
\frac{K_{\| 2}^{*}}{K_{\| 1}^{*}}=-\frac{\delta I_{\mathrm{ax} 1 d}}{\delta I_{\mathrm{ax} 2 d}} \triangleq-\alpha_{I d},
$$

which stands for a counterpart of the condition (43) where the influence of the electric field is replaced by the measured currents. For instance, for our 3-electrode probe and with $d=$ $(L / 2)+0.11 \mathrm{~m}$, one finds $\alpha_{I d}=1.23$. As expected, this process is equivalent to this based on (54) and (58), but has the advantage of being more straightforward. In the same way, one can directly fulfill the conditions (50) by imposing $K_{\theta 1}^{*}=0$ and $K_{\theta 2}^{*}>0$ in (59). Using these considerations, our experimented model of the emulated restoring forces takes the general form:

$$
\left(\begin{array}{c}
F_{r \|} \\
F_{r \perp} \\
M_{r}
\end{array}\right)=\left(\begin{array}{c}
K_{\| 1}^{*}\left(\delta I_{\mathrm{ax} 1}-\alpha_{I d} \delta I_{\mathrm{ax} 2}\right) \\
K_{\perp 1} \delta I_{\text {lat } 1}+K_{\perp 2} \delta I_{\mathrm{lat} 2} \\
K_{\theta 2}^{*} \delta I_{\text {lat2 }}
\end{array}\right) .
$$

Now, in order that (61) emulates two Coulomb charges (and realizes the counterpart of (53)), the lateral gains $K_{\perp 1}$ and $K_{\perp 2}$ need to satisfy the further relation deduced from (37), (40) and (60):

$$
\left(\begin{array}{c}
K_{\perp 1} \\
K_{\perp 2}
\end{array}\right)=\left(\begin{array}{cc}
A_{\perp 1} & 0 \\
0 & A_{\perp 2}
\end{array}\right)\left(\begin{array}{cc}
A_{\| 11} & A_{\| 21} \\
A_{\| 12} & A_{\| 22}
\end{array}\right)^{-1}\left(\begin{array}{c}
1 \\
-\alpha_{I d}
\end{array}\right) K_{\| 1}^{*} \triangleq\left(\begin{array}{c}
\nu_{1} \\
\nu_{2}
\end{array}\right) K_{\| 1}^{*} .
$$

Note that this relation is the only one required by the approximated model of the axial electric field of section 6.4. Moreover, it only depends on the intrinsic morphology of the probe and of the direct measurement of $\alpha_{I_{d}}$. For our 3-electrode probe, one introduces $\alpha_{I d}=1.23$ and (29) into (62), which gives:

$$
\left(\begin{array}{l}
\nu_{1} \\
\nu_{2}
\end{array}\right)=\left(\begin{array}{c}
+5.5 \\
-0.7
\end{array}\right)
$$

To conclude this section, introducing (62) into (61) yields:

$$
\left(\begin{array}{c}
F_{r \|} \\
F_{r \perp} \\
M_{r}
\end{array}\right)=K\left(\begin{array}{c}
\delta I_{\mathrm{ax} 1}-\alpha_{I d} \delta I_{\mathrm{ax} 2} \\
\nu_{1} \delta I_{\mathrm{lat} 1}+\nu_{2} \delta I_{\mathrm{lat} 2} \\
\xi \delta I_{\mathrm{lat} 2}
\end{array}\right) .
$$

where $\xi=K_{\theta 2}^{*} / K_{\| 1}^{*}$ and $K_{\| 1}^{*}=K$ stand for the angular reactivity and a scaling force factor respectively. In all the experiments hereafter reported, we use (64) with (63), while the two independent gains $K$ and $\xi$ are tuned in situ in a unique preliminary phase.

\section{$9 \quad$ Haptic feedback control law}

Though having the advantage of being intrinsically passive, the position-position control that we here adopt, suffers from several drawbacks. First, compared to the force-position law, it provides TS with low transparency, a limitation which is here compensated by using an extremely back-drivable master arm. Second, with small displacements of the master manipulator, we wish the slave to perform both long range displacements of low accuracy, and accurate fine maneuvers, which is difficult due to the size difference between our two work-spaces. To address these different issues, one commonly uses position and force adaptative scale factors (see Figure 11), online decoupling (see paragraph 9.3), a velocity sphere (Salcudean et al. 1995), or a combination of all these solutions. 


\subsection{Linearization of the slave and master Cartesian dynamics}

The Cartesian position-position control is conceptually simple and partly inspired from the active stiffness control (Salisbury 1980), or impedance control (Hogan 1985). To design such a controller, the idea consists of coupling with virtual springs and dampers, the dynamics of the master and the slave robots, both being considered as admittances in their respective Cartesian space. In the case of the slave robot, the Cartesian space has been already defined as the space of poses ${ }^{s} g_{p} \in S E(2)$ or alternatively the parametric space of $X_{s}=(x, y, \theta)^{T}$. As regards the master robot, i.e., the haptic interface, its roll, pitch and altitude DoFs being locked, the configuration space of the joystick frame $\mathcal{F}_{m}$ is also $S E(2)$ and its poses with respect to the interface basis are parameterized by the Cartesian coordinates vectors $X_{m}=\left(x_{m}, y_{m}, \theta_{m}\right)^{T}$. With these notations, the Cartesian dynamics of the master and slave are of the general form ${ }^{3}$ :

$$
M_{k}\left(q_{k}\right) \ddot{X}_{k}+C_{k}\left(q_{k}, \dot{q}_{k}\right)+Q_{k}\left(q_{k}\right)=J_{k}^{-T}\left(q_{k}\right) \tau_{k}+{ }^{s^{\star}} \mathbb{F}_{e x t ~},
$$

where the index $k$ stands for the slave or master index $m$ or $s$, while $M_{k}$ and $C_{k}$ are the Cartesian mass matrix and the Cartesian vector of Coriolis-centrifugal forces, $q_{k}$ and $\tau_{k}$ are the vectors of joint angles and torques, $J_{k}$ is the Jacobian matrix of the manipulator, and $s^{\star} \mathbb{F}_{\text {ext } k}$ stands for the wrench of external Cartesian forces applied onto the considered manipulator. In the case of the master, ${ }^{s^{\star}} \mathbb{F}_{\text {ext } m}$ is exerted by the operator (on the joystick) and denoted ${ }^{\star} \mathbb{F}_{o}$, while in the case of the slave ${ }^{s^{\star}} \mathbb{F}_{\text {ext } s}$ stands for our emulated electric restoring wrench ${ }^{s^{\star}} \mathbb{F}_{r}$. Finally, $J_{k}^{-T} \tau_{k}=s^{\star} \mathbb{F}_{k}$, $k=m, s$, stands for the wrenches coupling the master and the slave. Their design for impedance control is briefly summarized hereafter.

To perform impedance (linear) control, one can easily resort to some simplifications allowing removing most of the nonlinearities of the general dynamics (65). In fact, the slave is a Cartesian robot, and one can consider that $C_{s}=Q_{s}=0$, while $J_{s}$ and $M_{s}$ are some constant diagonal matrices depending on the mechanical design of the gantry. As regards the master, this is a light parallel robot subject to motions of small magnitude. Thus, one can also neglect the Coriolis forces $C_{m}$ as well as the inertia of the legs, and consider in $M_{m}$ and $Q_{m}$, the influence of the joystick only. In such simplified conditions, one can consider the so-called Jacobian scheme:

$$
\tau_{m}=J_{m}^{T}\left({ }^{\star} \mathbb{F}_{m}+Q_{m}\right), \tau_{s}=J_{s}^{T}\left(s^{\star} \mathbb{F}_{s}\right),
$$

which once applied to the two robots, allows changing their Cartesian dynamics (65) into the linearized-decoupled ones:

$$
M_{m} \ddot{X}_{m}={ }^{s^{\star}} \mathbb{F}_{o}+{ }^{s^{\star}} \mathbb{F}_{m}, M_{s} \ddot{X}_{s}={ }^{s^{\star}} \mathbb{F}_{s}+{ }^{s^{\star}} \mathbb{F}_{r},
$$

where ${ }^{s^{\star}} \mathbb{F}_{m}$ and ${ }^{s^{\star}} \mathbb{F}_{s}$ define a feedback control law emulating the expected position-position dynamics of the remote control loop as detailed in the next section. Finally, it is worth noting that the mapping $\left(s^{\star} \mathbb{F}, s^{\star} \mathbb{V}\right) \mapsto(\tau, \dot{q})$ being lossless, the law (66) preserves the intrinsic passivity of the position-position scheme.

\subsection{Position-position control loop}

The principle of the position-position control consists of coupling together the master and slave linearized dynamics (67) with a law of the form:

$$
{ }^{s^{\star}} \mathbb{F}_{m}={ }^{s^{\star}} \mathbb{K}_{m}\left(X_{s}-X_{m}\right)+{ }^{s^{\star}} \mathbb{B}_{m}\left(\dot{X}_{s}-\dot{X}_{m}\right),{ }^{s^{\star}} \mathbb{F}_{s}={ }^{s^{\star}} \mathbb{K}_{s}\left(X_{m}-X_{s}\right)+{ }^{s^{\star}} \mathbb{B}_{s}\left(\dot{X}_{m}-\dot{X}_{s}\right),
$$

\footnotetext{
${ }^{3}$ Note that this form is shared by serial and parallel robots.
} 
where from left to right, the first of the above relations stands for a PD servoing of the master on the slave position while the second relation stands for a PD servoing of the slave position on the master one. Furthermore, taking $s^{\star} \mathbb{K}_{m}={ }^{s^{\star}} \mathbb{K}_{s}=\mathbb{K}$ and ${ }^{s^{\star}} \mathbb{B}_{m}={ }^{s^{\star}} \mathbb{B}_{s}=\mathbb{B}$, allows emulating a mechanical spring-damper coupling, which is intrinsically passive. In such conditions, ${ }^{*} \mathbb{F}_{m}=-s^{\star} \mathbb{F}_{s}$ and (67) is changed into:

$$
M_{m} \ddot{X}_{m}+\mathbb{B}\left(\dot{X}_{m}-\dot{X}_{s}\right)+\mathbb{K}\left(X_{m}-X_{s}\right)={ }^{s^{\star}} \mathbb{F}_{o}, M_{s} \ddot{X}_{s}+\mathbb{B}\left(\dot{X}_{s}-\dot{X}_{m}\right)+\mathbb{K}\left(X_{s}-X_{m}\right)={ }^{s^{\star}} \mathbb{F}_{r} .
$$

Finally, these two linear decoupled dynamics define the master and slave admittance $Y_{m}$ and $Y_{s}$ of figure 11 .

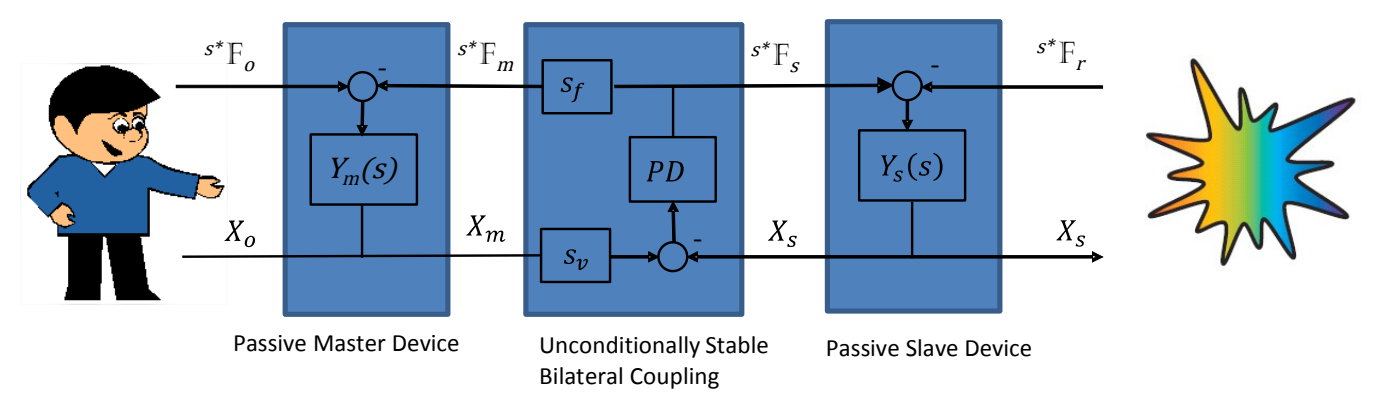

Figure 11: Implemented position-position Teleoperating System (TS). $s_{v}$ and $s_{f}$ are matrices of scaling "velocity" and "force" factors.

\subsection{Switching between coupled and uncoupled position control modes}

According to the position-position scheme, when the operator moves the joystick in the master space, the probe is moved with a similar motion but amplified by a certain scaling factor. Each of these two motions is defined with respect to a reference frame fixed in its own space, these two frames $\mathcal{F}_{m}$ and $\mathcal{F}_{s}$ being matched to each other. Due to the difference of size between the two working spaces (slave and master) and the limitations of the scaling factor (imposed by the accuracy), this frame matching needs to be updated. This is especially the case when performing long range motions in the slave space. This updating process works as follows. At any given time $t_{0}$, if the operator pushes a button, the two frames $\mathcal{F}_{p}\left(t_{0}\right)$ and $\mathcal{F}_{m}\left(t_{0}\right)$ are matched to each other, and $\mathcal{F}_{p}\left(t_{0}\right)$ becomes the new slave reference frame $\mathcal{F}_{s}$ (i.e. the time-evolution $\mathcal{F}_{m}(t) / \mathcal{F}_{m}\left(t_{0}\right)$ for $t>t_{0}$, generates a homothetic motion of $\left.\mathcal{F}_{p}(t) / \mathcal{F}_{p}\left(t_{0}\right)\right)$. Reciprocally, when the joystick reaches the boundary of its working space (defined as a ball in the space of $X_{m}$ centered on the joystick neutral position), the operator can switch off the frame matching. Then, the slave is locked in its current position while the joystick being uncoupled from the slave, it can be moved freely by the operator in a novel reference frame position fixed by the next activation of the button. This iterative process can be repeated as many times as required by the task. We now illustrate it on an arbitrary path specified in the master space by the 6 maneuvers (labelled in chronological order $A, B, . . F$ ) displayed in figure $12(\mathrm{a})$, and reproduced in the slave one as shown in figure 12(b). In this test, the electric force is not fed-back to the master, while as in all the subsequent experiments, the working space is such that $-2<\sqrt{x_{m}^{2}+y_{m}^{2}}<2 \mathrm{~cm}$ and $-10<\theta_{m}<10^{\circ}$. As expected, when the joystick reaches the limit of the working ball (drawn by a circle), the operator switches off 
the matching between two spaces, and then moves the master to another pose which becomes the reference frame for the next manoeuver. In the slave space, the path of the probe appears as the iterative cumulation (integration) of all these elementary paths, each being constructed with respect to a different reference frame $\mathcal{F}_{s}$ as indicated in figure 12(b).
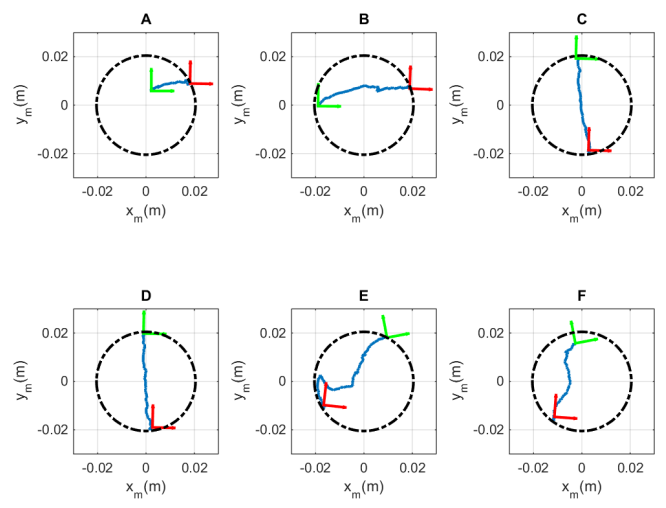

(a)

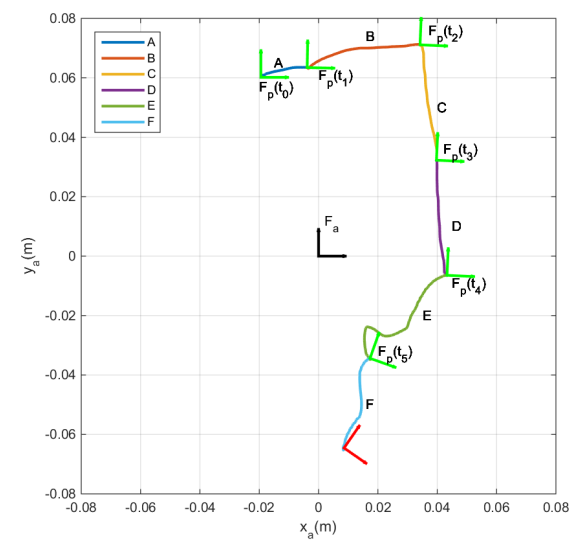

(b)

Figure 12: (a): Paths followed by the master. In green the initial frame of the manoeuver, in red the final one. (b) Path in the slave space, with $\mathcal{F}_{a}$ an absolute frame located at the tank center, and $\mathcal{F}_{p}\left(t_{i}\right)$ the successive values of the reference frame $\mathcal{F}_{s}$, updated at the switching instants $t_{i}$ which bound the manoeuvres $A, B, C \ldots F$.

To supplement the illustration, figure 13(a) shows the time-evolution of the joystick pose parameters $X_{m}=\left(x_{m}, y_{m}, \theta_{m}\right)^{T}$ and of those of the probe frame w.r.t. an absolute slave frame $\mathcal{F}_{a}$ positioned at the center of the tank. This vector of parameters is denoted $X_{a}=\left(x_{a}, y_{a}, \theta_{a}\right)^{T}$, with "a" for "absolute". To illustrate the frame updating, the time-evolution of a switching Boolean variable ( $B=1$ if the master and slave are coupled, and $B=0$ if not) is plotted in figure 13(b). These further plots show how $X_{m}$ moves between two manoeuvres (coupled phases), while in these cases, $X_{a}$ is locked in its last coupled position.

\section{Experimental characterization of the electro-haptic loop}

This section deals with the implementation and characterization of the haptic loop. This is the preliminary step before its operational experimentation that will be addressed in the next section. In this section, we consider a test where the joystick remains in its working ball with no change of reference frame.

\subsection{Experimental implementation of the Teleoperation System (TS)}

In all tests, we uses $s_{v}=\operatorname{diag}(1,1,1), s_{f}=\operatorname{diag}(0.3,0.3,0.01)$ which are the matrices scaling the velocities and forces between the master and slave spaces. In practise, the PD controller of figure 11 is reduced to a single diagonal proportional component, with gains defined by:

$$
K_{p_{x}}=100 \mathrm{~N} / \mathrm{m}, K_{p_{y}}=100 \mathrm{~N} / \mathrm{m}, K_{p_{\theta}}=30 \mathrm{Nm} / \mathrm{rad},
$$



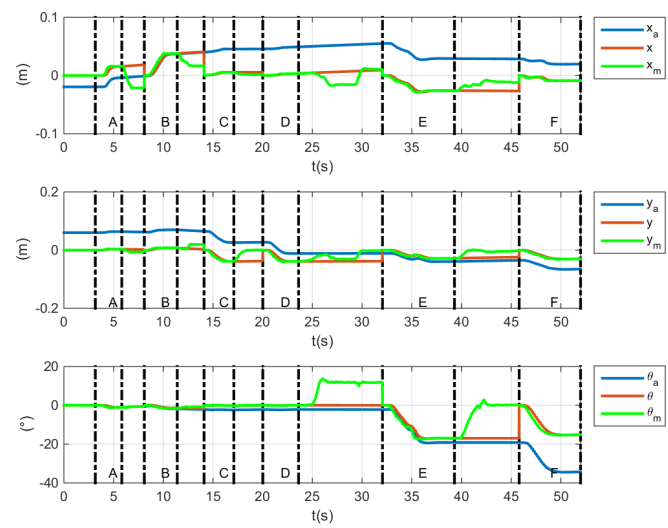

(a)

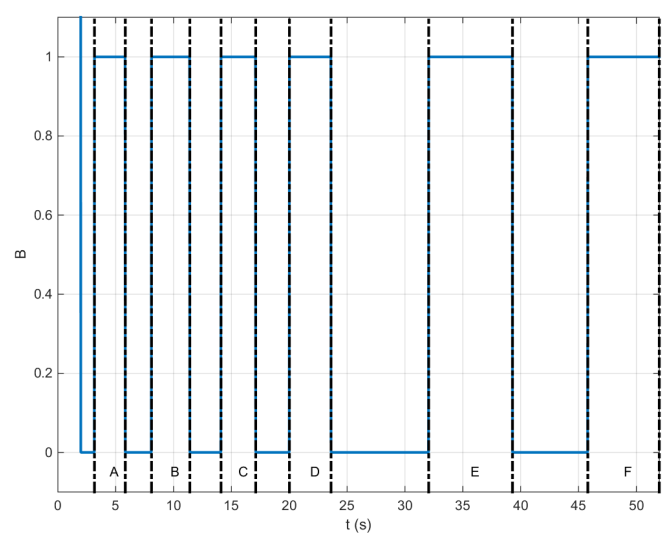

(b)

Figure 13: (a) Time evolution of $X_{m}$ (red), $X_{s}$ (green) and $X_{a}$ (blue) in the same conditions as figure (12). (b) Time evolution of the switching Boolean variable $B$ for the same example.

while the scaling factor and the angular reactivity of the electric restoring wrench (64) are

$$
K=5000 \mathrm{~N} / \mathrm{A}, K \xi=5000 \mathrm{Nm} / \mathrm{A} .
$$

Note that this set of gains has been chosen (by trials and errors) in order to ensure the stability of the slave-master and master-slave position loops in a comfortable bandwidth for the operator ${ }^{4}$.

\subsection{Characterization based on the alternation of single DoF com- mands}

In this first test, the probe is initially positioned in one of its desired poses at the middle of one of the walls of the tank presented in section 2.2. Then, the test starts by a phase from $t \simeq 4 \mathrm{~s}$ to $t \simeq 30$ s during which $x_{m}$ is moved, then from $t \simeq 30$ s to $t \simeq 58, y_{m}$ is moved, and finally $\theta_{m}$ is moved from $t \simeq 58 \mathrm{~s}$ to $t \simeq 95 \mathrm{~s}$. For the operator's comfort, the coupling wrench ${ }^{s^{\star}} \mathbb{F}_{m}=-s^{\star} \mathbb{F}_{s}$ is not fed back to the joystick but solely monitored by the teleoperation system.

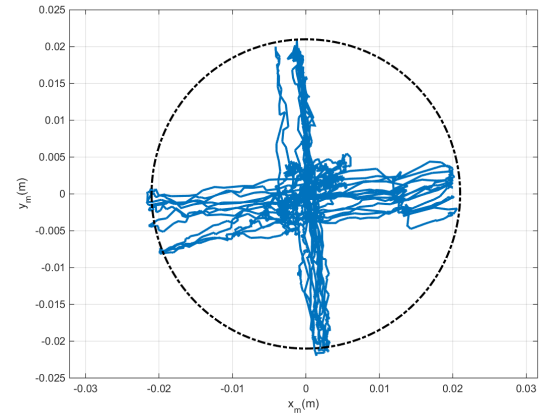

(a)

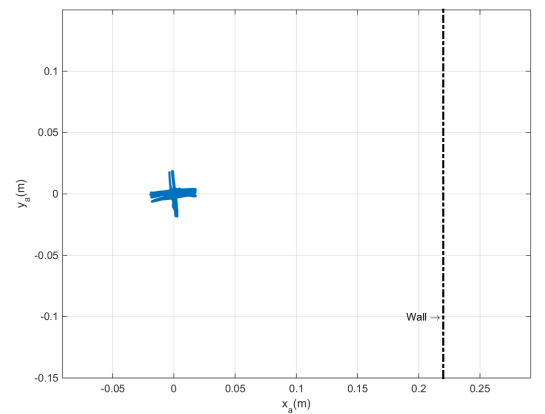

(b)

Figure 14: Path of the joystick (a) and the probe (b) in their Cartesian space: when the $x, y$ and $\theta$ DoFs are moved one after the other.

\footnotetext{
${ }^{4}$ Note here that though being unconditionally stable, the position-position coupling can be destabilized by the inherent delays introduced by digital transmissions (Hannaford \& Ryu 2002).
} 

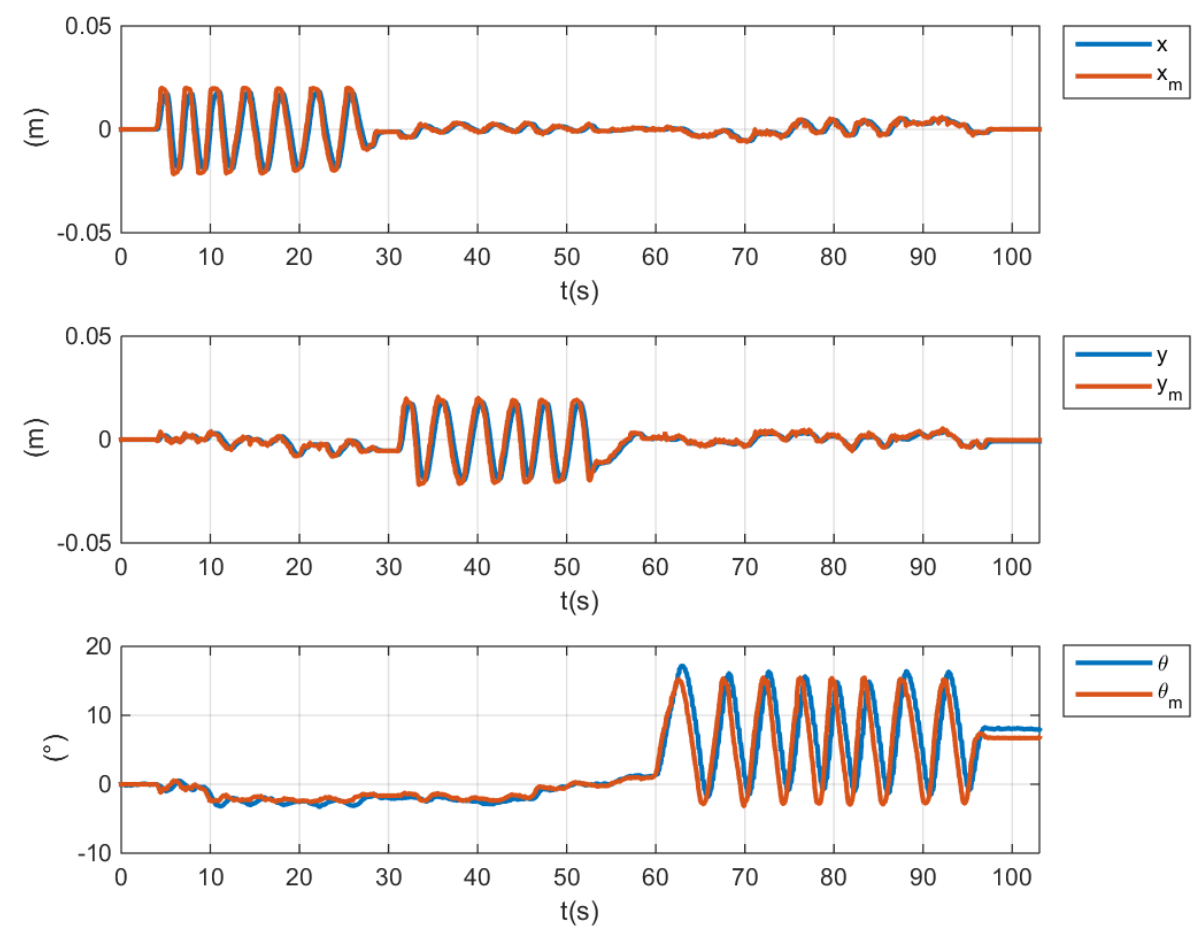

Figure 15: Time evolution of $X_{m}$ and $X_{s}$ when the $x, y$ and $\theta$ DoFs are moved one after the other.
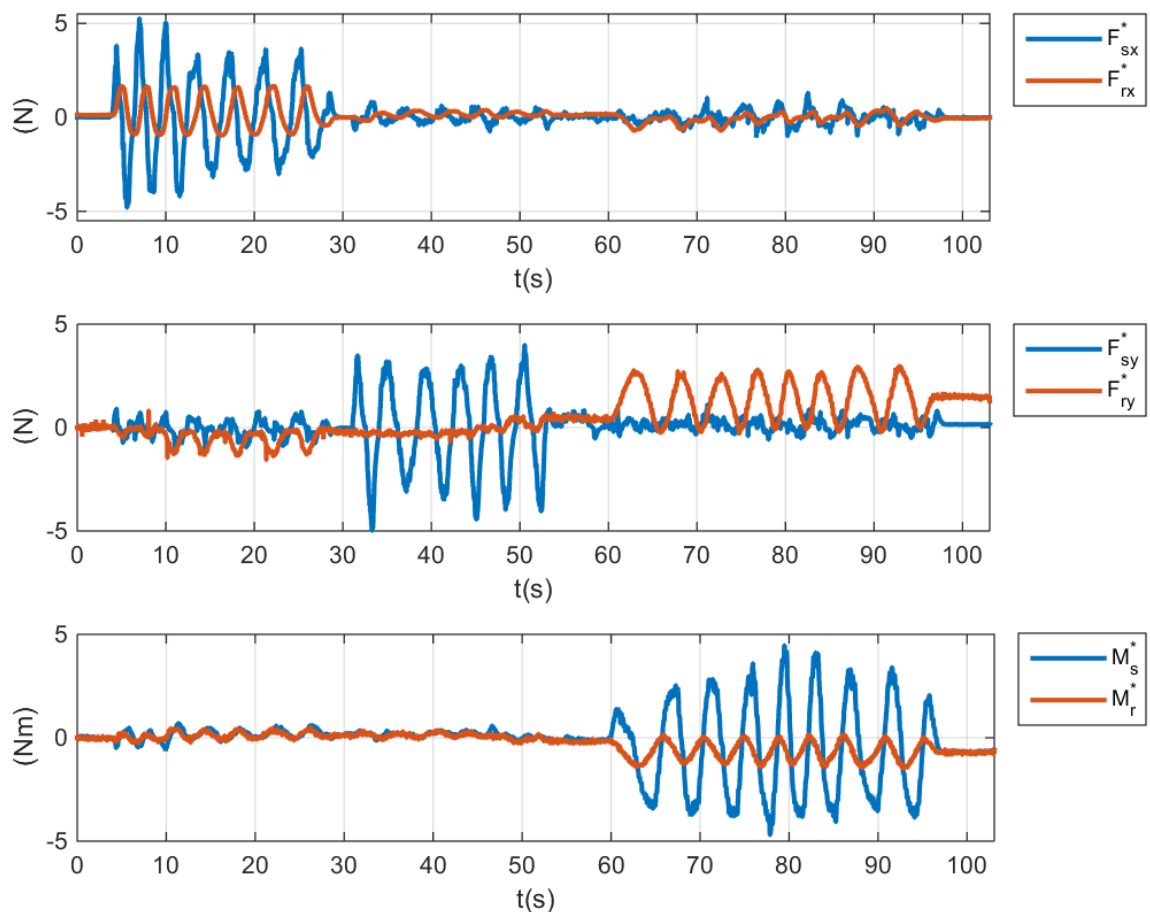

Figure 16: Time evolution of $s^{\star} \mathbb{F}_{s}$ and ${ }^{s^{\star}} \mathbb{F}_{r}$ when the $x, y$ and $\theta$ DoFs are moved one after the other. 
The paths of $X_{m}$ and $X_{s}$ are displayed in figures 14-a and 14-b, while figure 15 displays the time evolution of their three components. The time evolution of the three components of the positionposition coupling wrench ${ }^{s^{\star}} \mathbb{F}_{s}=-{ }^{s^{\star}} \mathbb{F}_{m}=\left(F_{s x}^{\star}, F_{s y}^{\star}, M_{s}^{\star}\right)^{T}$ and of the electric wrench $s^{\star} \mathbb{F}_{r}=$ $\left(F_{r x}^{\star}, F_{r y}^{\star}, M_{r}^{\star}\right)^{T}$ are reported in figures 16. As expected, both the positions $X_{m}=\left(x_{m}, y_{m}, \theta_{m}\right)^{T}$ and $X_{s}=(x, y, \theta)^{T}$ follow each other. The examination of the time lags between the curves of 15 and 16 allows confirming the causality of the events. In the case of the $x$-channel, the first cause is the $x_{m}$-motion which contracts the spring of the position-position coupling wrench (its $x$ component $F_{s x}^{\star}$ ). This wrench makes the slave move along $x$ so generating an electric force $F_{r x}^{\star}$ which tends to balance the spring force along $x$. The same causality holds along the $y$ and $\theta$ DoFs. However, in these further cases, due to the $\theta-y$ couplings introduced by the model of electric forces (64), any $\theta$-variation of the probe generates an additional restoring electric force $F_{r y}^{\star}$. Finally, the probe being perpendicular to the wall, this coupling does not express on the $y$-channel, and the $y$-variations do not generate any restoring lateral force $F_{r y}^{\star}$.

\subsection{Experimental characterization of passivity}

The passivity of the model of electric forces has been theoretically proved in section 7.5. One can now confirm this prediction experimentally. To that end, we reconsider the single DoF test of section 10.2 and numerically calculate from (56), the values of the $S$ function along the probe path. This time evolution of $S$ is displayed in figure 17-a. As this has been observed for all our experiments, the path integral remains lower bounded by zero. To supplement this observation, we reported in figure 17-b, the evolution of this storing function $S$ versus the $x$-coordinate between times $t=4 \mathrm{~s}$ and $t=30 \mathrm{~s}$, i.e. along a few cycles of the first phase of the test of section 10.2 (when the $x$-DoF is moved). As predicted by the theoretical analysis of section 5 , in this case the integral nearly draws the typical profile of the Lennard-Johns interaction potential close to its equilibrium position, the slight discrepancies being due to the residual motions of the two other DoF that the operator cannot avoid.

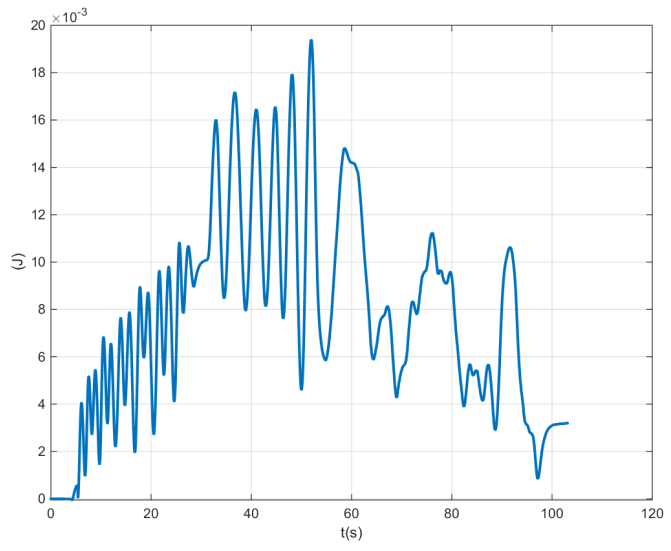

(a)

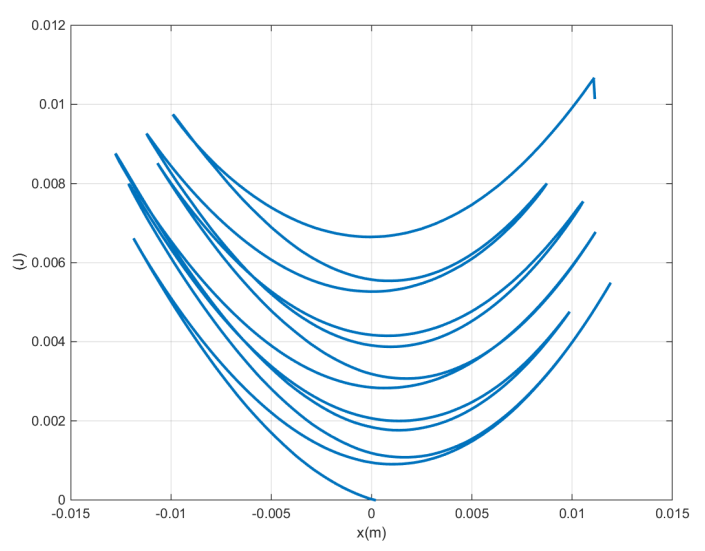

(b)

Figure 17: (a): Time evolution of the storing function $S$ along the entire test of section 10.2. (b): $S$ versus $x$ in the first phase of the test of section 10.2, i.e., when $x$ is moved. 


\section{Operational tests of the electro-haptic feedback loop}

In this section, our model of electric restoring forces is used in the remote-control position-position architecture of section 9 . The concept of the electro-haptic feedback loop has been tested through a set of experiments carried out by an operator in different working conditions. We here report a representative sample of these first results and give an interpretation of them.

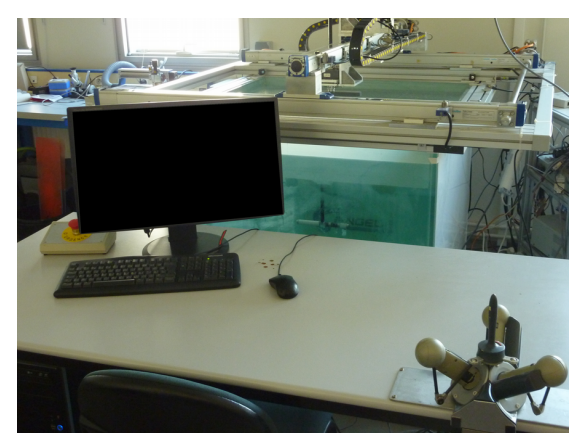

(a)

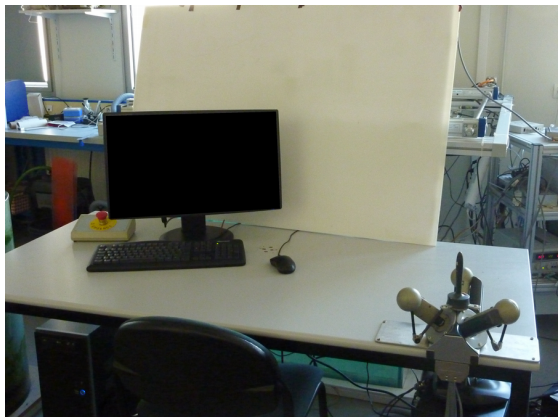

(b)

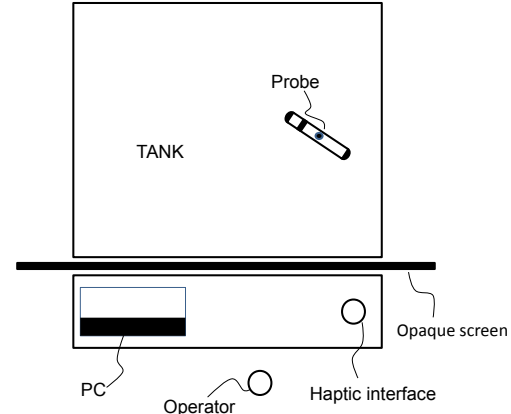

(c)

Figure 18: Operational view point without (a) and with (b) the opaque screen. (b): Top-view schematize of the setup.

\subsection{Experimental results}

The task consists in following the boundaries of the environment while the probe is maintained perpendicular to them at a fixed distance. The subjective goal of the operator is to push the probe as a far as possible along the translational DoF offering zero force, while on the two others, he allows himself to be guided by some restoring force and torque. These experiments consist of four tests numbered 1, 2, 3, 4 and organized as follows. In the first test, the probe is alone in the tank of section 2.2. In the second, we add a small insulating sphere close to one of its walls. In the third, a conductive spherical object is added in the scene. Finally, in the fourth test, some movable walls are added in order to create a "hole" in a wall. Note that, compared to our ideal infinite wall, all these tests introduce some electric perturbations: corners, hole, objects that we expect to be rejected by our remote-control strategy. All the tests are started in the same initial conditions. The probe is first positioned in its equilibrium position at the middle of one wall. The joystick is positioned close to its neutral position and its frame is matched with the probe frame in the equilibrium position. All the tests are performed with no visual feedback since the tank is occulted with an opaque screen (see figure 18). The results are presented as follows. For the 4 tests, we reported the paths of the probe in the tank (figures 20, 23, 26, 29); the time-evolution of $X_{m}=\left(x_{m}, y_{m}, \theta_{m}\right)^{T}$ and $X_{a}=\left(x_{a}, y_{a}, \theta_{a}\right)^{T}$ (figures 21, 24, 27, 30); the time evolution of ${ }^{p} \mathbb{F}_{r}=\left(F_{r x}, F_{r y}, M_{r}\right)^{T}$ (figures 22, 25, 28, 31). Note that $X_{a}$ and ${ }^{p} \mathbb{F}_{r}$ are reconstructed from $X_{s}$ and ${ }^{s^{*}} \mathbb{F}_{r}$ with the frame updating process of section 9.3 and using equation (46). These variables are preferred to $X_{s}$ and ${ }^{\star} \mathbb{F}_{r}$ for the convenience of interpretation of results. To initiate our reporting, the test 1 is enriched of the plots of the joystick path in the master space and the time-evolution of the Boolean variables indicating the switches between coupled pairs of reference frames in the two spaces (figures 19(a) and 19(b)). For the purpose of readability, we used a color code in the above plots. In figures $21,24,27,30$, the $X_{a}$ and $X_{m}$ plots are indicated with the blue and red colors respectively. The green color is preferentially used to indicate the values of some 
variables when the distance between the probe and the nearest orthogonal wall is equal to the expected value $d$. Finally, along the probe paths of figure 20, 23, 26, 29 several snapshots of its pose are indicated in dashed line. On the corresponding sub-figures numbered (a) these snapshots are plotted at a regular time rate, while in sub-figures (b), they are plotted at key instants relevant to our discussion. All these snapshots are indicated by capital letters $(A, B, C \ldots)$ that are used in other plots to point out the corresponding instants.

\subsection{Test 1}

In this first test, the operator goes around the tank in 900s while maintaining the probe perpendicular along the 4 walls. To perform the task, the operator has updated the reference slave frame 85 times according to the plots of figures 19(a) and 19(b). As illustrated in figures 20(b), the probe correctly performs the task through a sequence of distinguishable phases consisting of translations along the walls $(A \rightarrow B, C \rightarrow D, E \rightarrow F, G \rightarrow H, I \rightarrow J)$ and rotation around corners $(B \rightarrow C$, $D \rightarrow E, F \rightarrow G, H \rightarrow I)$. Along translation phases, the plots of figure 21 clearly show that one of the probe positional DoF varies linearly, while the two others are maintained fixed. Reciprocally, on the rotating phases, only the angular DoF linearly varies. Looking at the plots of figure 22 shows that over the translation phases, the operator feels a zero restoring force $\left(F_{r \perp}\right)$ on the translated DoF. The same plot shows that the restoring force and torque, $F_{r \|}$ and $M_{r}$, fed back to the operator are small along translation phases but brutally vary in the rotation phases. In this case, referring to the method of mirror images in electrostatics (Jackson 1999), when the probe is close to a corner, it is no more influenced by a single mirror image (reflected by an infinite wall), but by three images, one per wall and another for the corner (Boyer et al. 2012). The superposition of all these images generates a reflected field rotated (along the diagonal of the corner) with respect to that generated by a single wall. In all these phases, the restoring momentum $M_{r}$ encourages the operator to align the probe with this rotated field while the two components of the restoring force $F_{r}$ keep the probe at a reasonable distance $(\simeq d)$ to the walls (see the three plots of figure 22, e.g. between $B$ and $C$ ). The combined effects of the three components of ${ }^{\star} \mathbb{F}_{r}$ are consistent together, and the operator has the feeling to escape a valley by performing a rotational manoeuver.

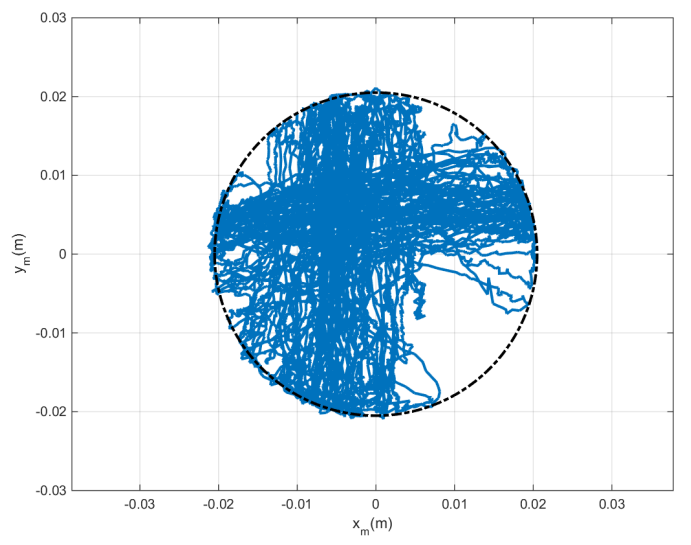

(a)

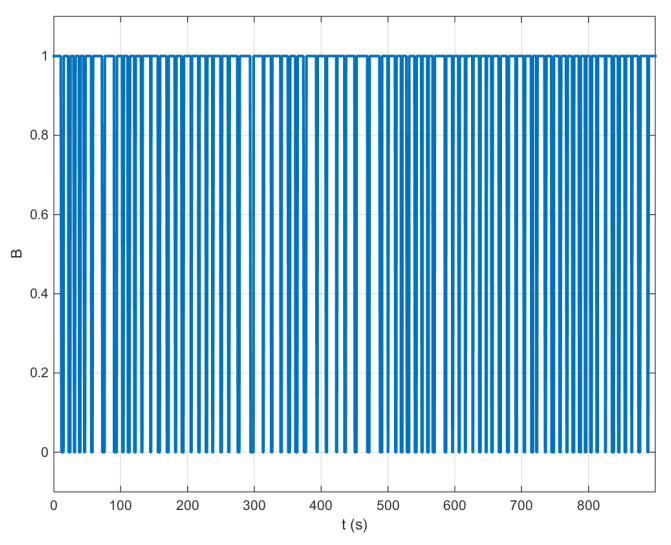

(b)

Figure 19: Test 1: Path of the joystick in the master space (a), and time evolution of $B$ (b). 


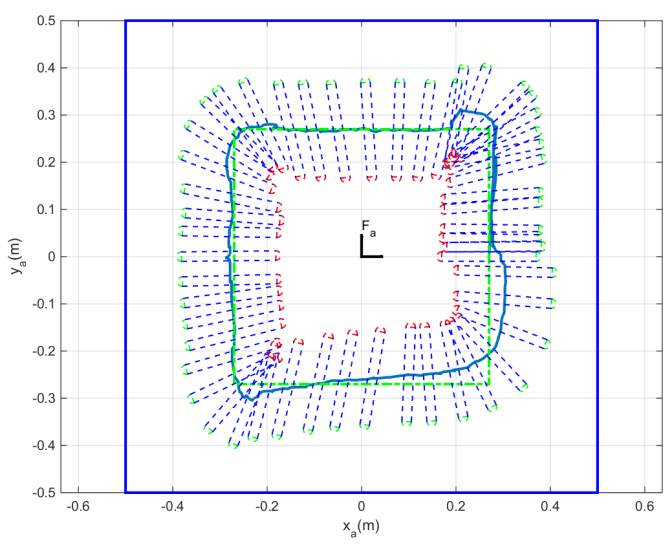

(a)

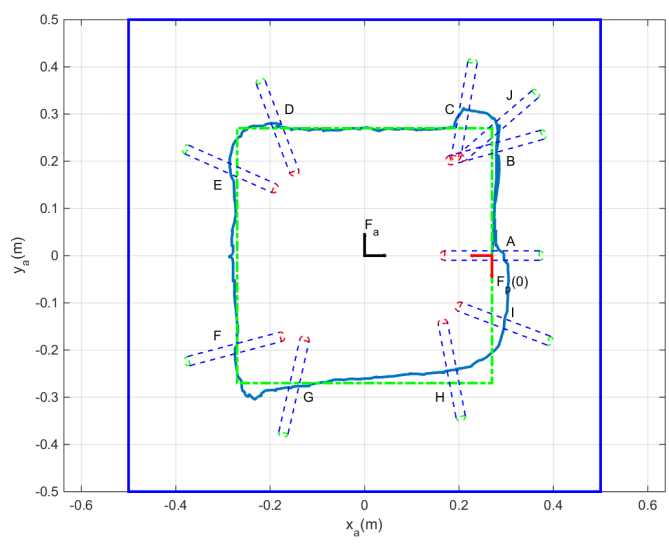

(b)

Figure 20: Test 1: Path of the probe in the slave space (a), with some distinguished poses (b).
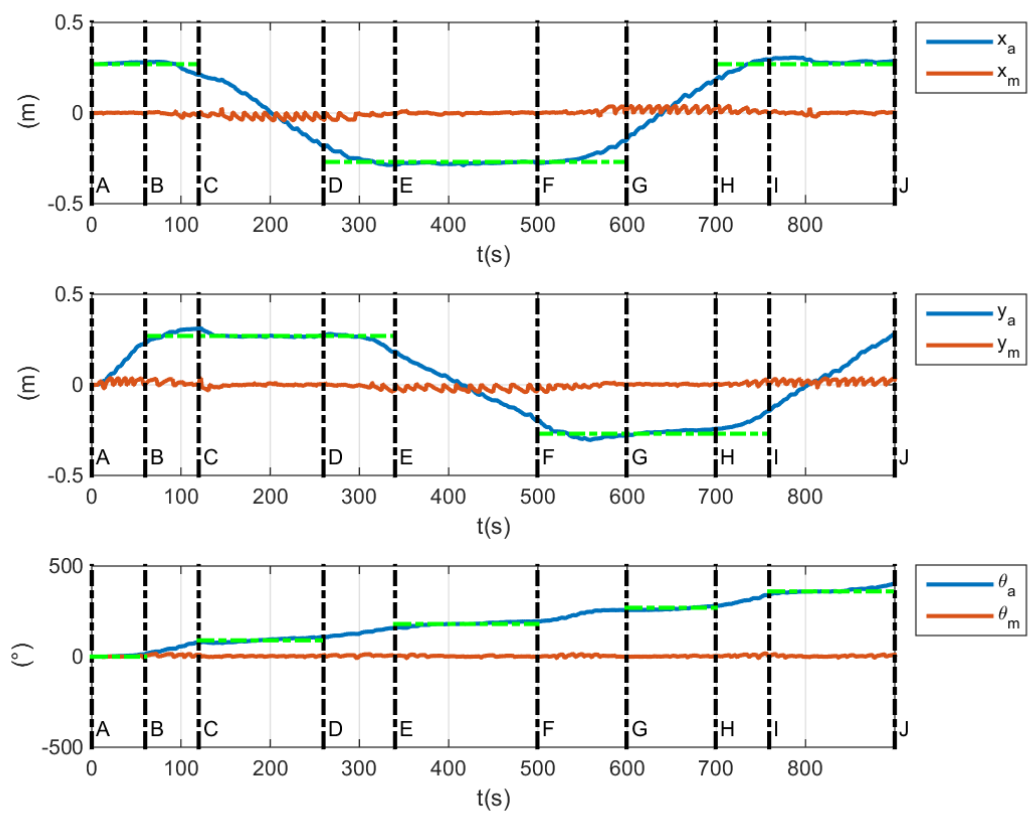

Figure 21: Test 1: Time evolution of $X_{m}$ and $X_{s}$. 

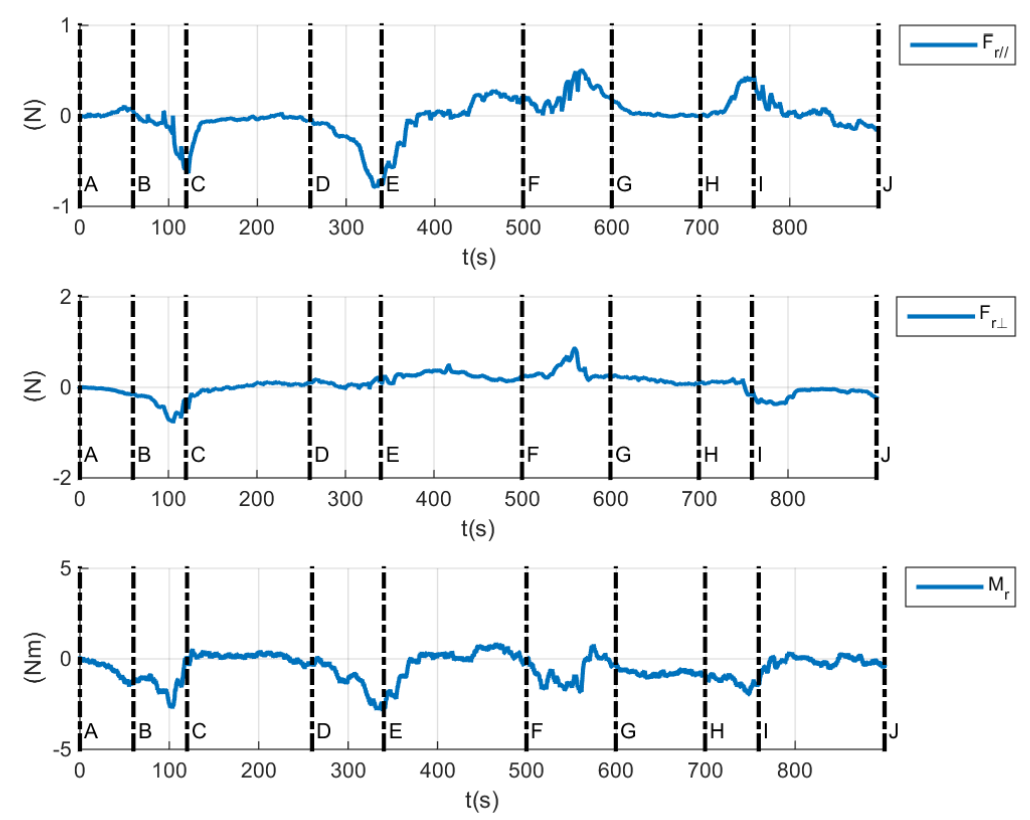

Figure 22: Test 1: Time evolution of ${ }^{p} \mathbb{F}_{r}$.

\subsection{Test 2}

In this second test, a $6 \mathrm{~cm}$ diameter sphere has been placed at a distance of $6 \mathrm{~cm}$ from one of the walls as indicated in figure 23(a). The sphere being insulating, for the probe it is electrically equivalent to a bump on the wall. The test starts as that of section 11.2 with a translation $A \rightarrow B$, a rotation at a corner $B \rightarrow C$, and another translation $C \rightarrow D$. Looking at the top plot of figure 25 shows that when approaching the sphere (after $D$ ), a repulsive lateral restoring force $F_{r \perp}$ is produced by the sphere on one side of the probe. After the probe has passed the object, a similar repulsive force is applied on it, but on the other side. At the same time, the probe is slightly pushed away (of about $3 \mathrm{~cm}$ ) from the wall between $D$ and $E$ by a force $F_{\|}$(see top plot of figure 25). These forces are fed to the operator who feels a negative (resistive) force followed by a positive (driving) force along $x_{m}$ and a negative one along $y_{m}$. Due to the $y-\theta$ couplings, figure 23(b) shows that this also generates two slight rotations of opposite sign before and after passing the sphere (note that this coupling does not express when the wall is alone as seen in section 10.2). Remarkably, the influence of the sphere remains perceptible in spite of the fact that its contribution to the reflected field is about 3 orders $\left(\approx 1 / d^{3}\right)$ lower than that reflected by the wall $(\approx 1 / d)$ (Boyer et al. 2012). At the end, the presence of the sphere generates on the joystick, a feeling of sweeping past a beading. Finally, beyond $E$, the operator achieves the task with a corner rotation $E \rightarrow F$ and a wall parallel translation $F \rightarrow G$. 


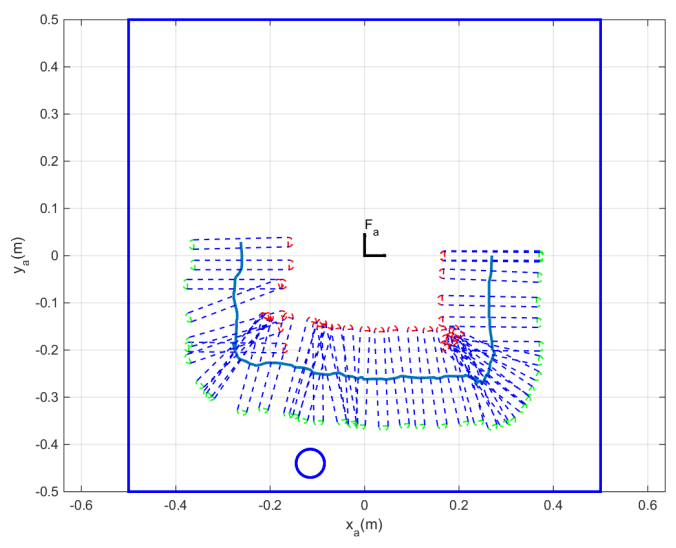

(a)

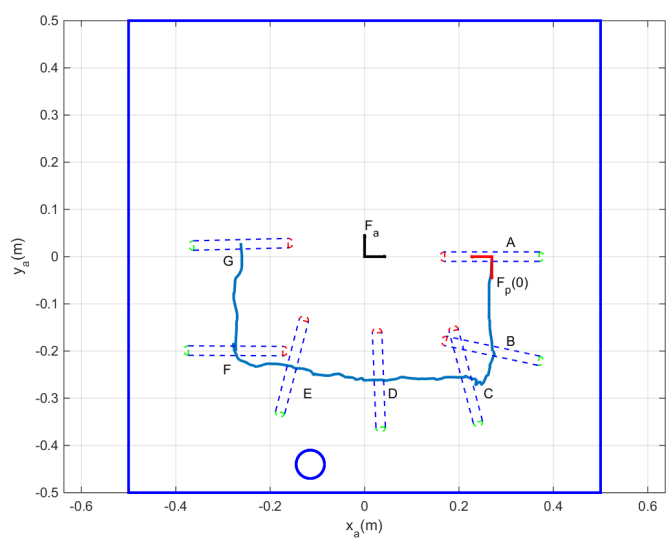

(b)

Figure 23: Test 2: Path of the probe in the slave space (a), with some distinguished poses (b).
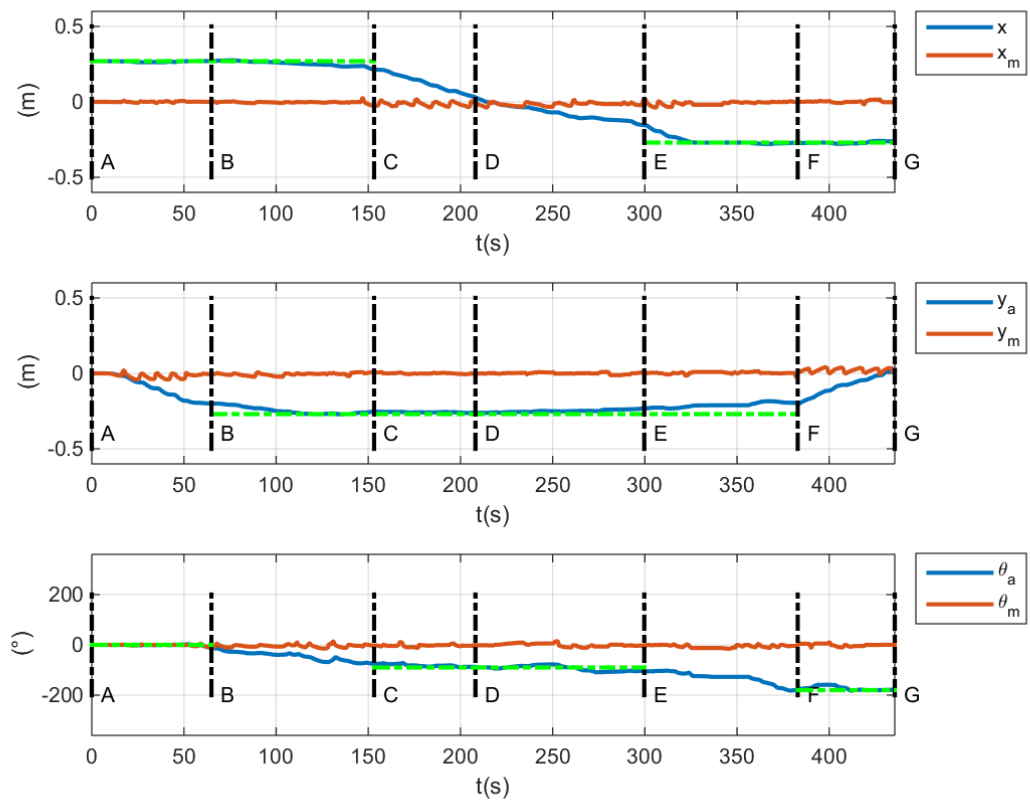

Figure 24: Test 2: Time evolution of $X_{m}$ and $X_{s}$. 

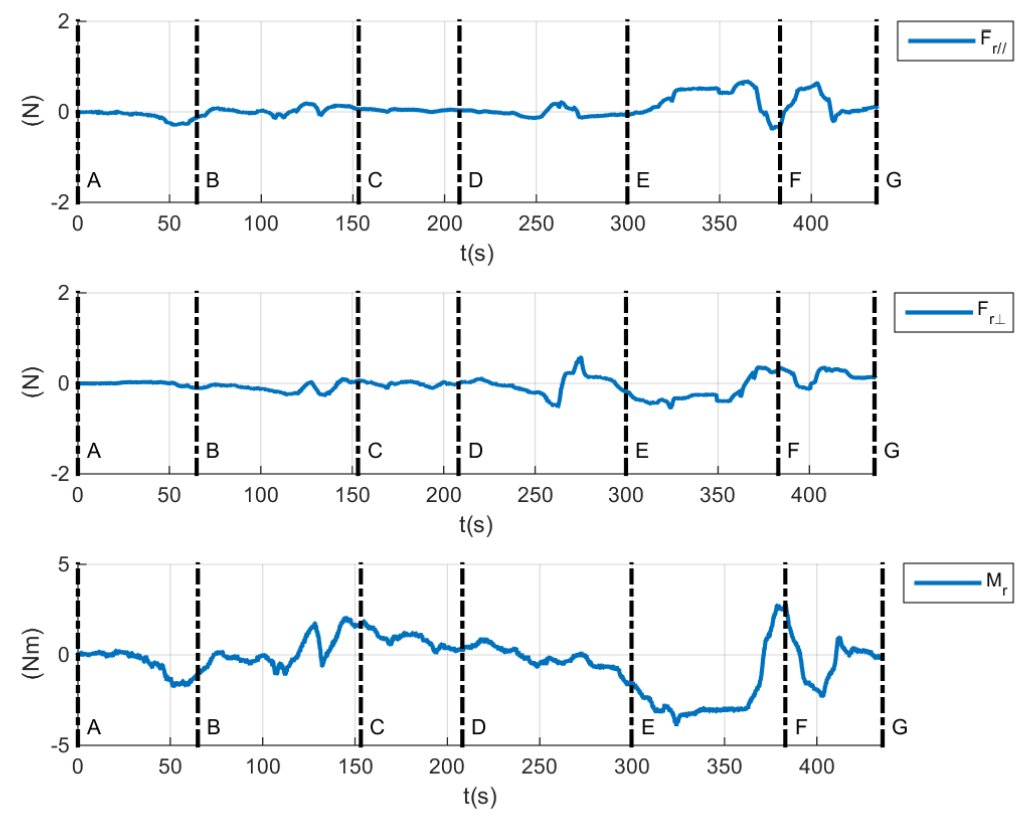

Figure 25: Test 2: Time evolution of ${ }^{p} \mathbb{F}_{r}$.

\subsection{Test 3}

In this test, a $6 \mathrm{~cm}$ diameter conductive sphere is placed at $10 \mathrm{~cm}$ from the wall as pictured in figure 26. The probe starts again in an equilibrium pose $A$ at the middle of one wall. The test includes two translation phases $A \rightarrow B$ and $C \rightarrow D$, and one corner rotation $B \rightarrow C$. As for an insulating object, the probe avoids the sphere while being pushed away from the wall beyond the desired distance $d$ (see $D \rightarrow F$ ). However, in this second case, the probe orientation strongly changes between $D$ and $F$ (see figure 26). Looking at the bottom plot of figure 28, this rotation is generated by the restoring momentum $M_{r}$ which quickly increases and decreases above and below zero between $D$ and $F$. This can be explained by the fact that when the probe approaches the sphere, the sphere is polarized by the probe field (and its mirror image reflected by the wall). As a result, an induced dipolar momentum parallel to the wall located on the sphere appears. This induced dipolar momentum and its mirror image generate together a further perturbative electric field nearly parallel to the wall, along which the probe tries to align (thanks to $M_{r}$ ). While the probe passes the sphere or its mirror image, its polarization is inverted which creates the changes of sign of $M_{r}$ between $D$ and $E$, and $E$ and $F$ on bottom plot of 28. Just after passing the sphere, the wall becomes dominant and the probe quickly reorients toward it and the next corner. Once again the operator feels a beading while sweeping past the conductive sphere, but this time on the angular DoF of the joystick. 


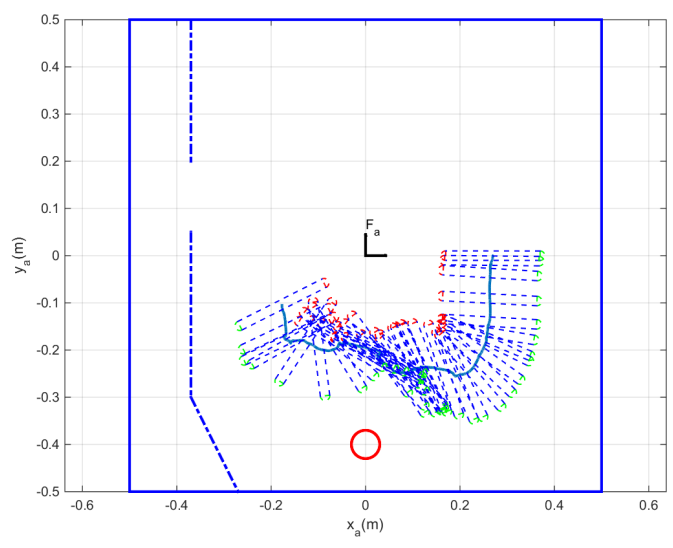

(a)

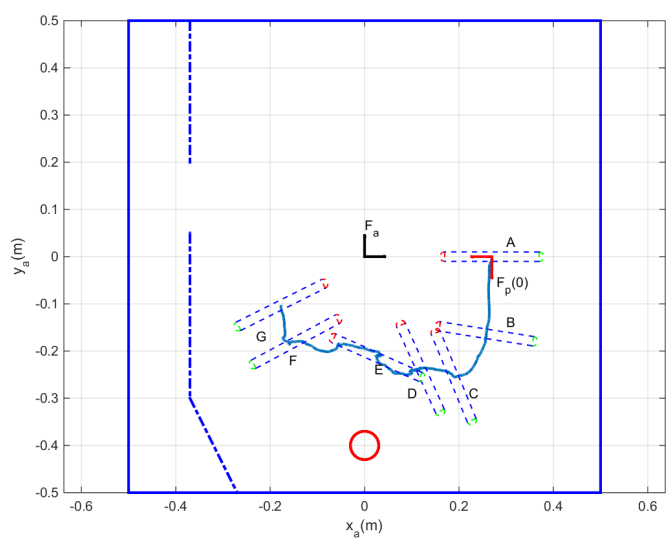

(b)

Figure 26: Test 3: Path of the probe in the slave space (a), with some distinguished poses (b).
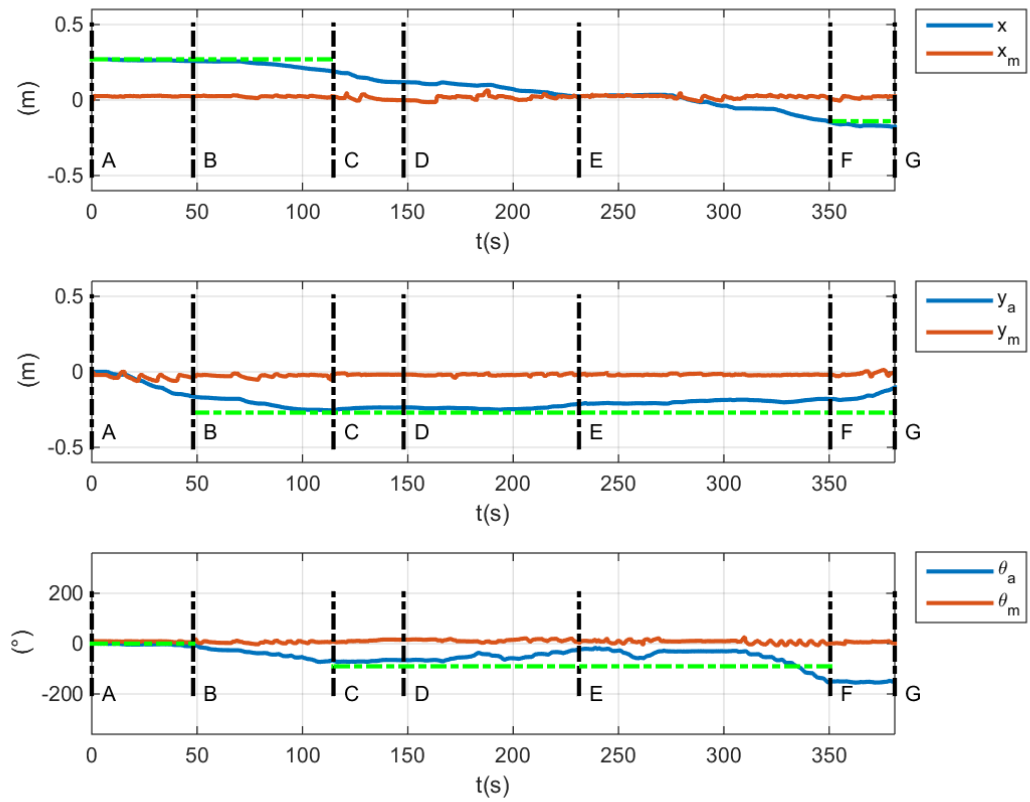

Figure 27: Test 3: Time evolution of $X_{m}$ and $X_{s}$. 

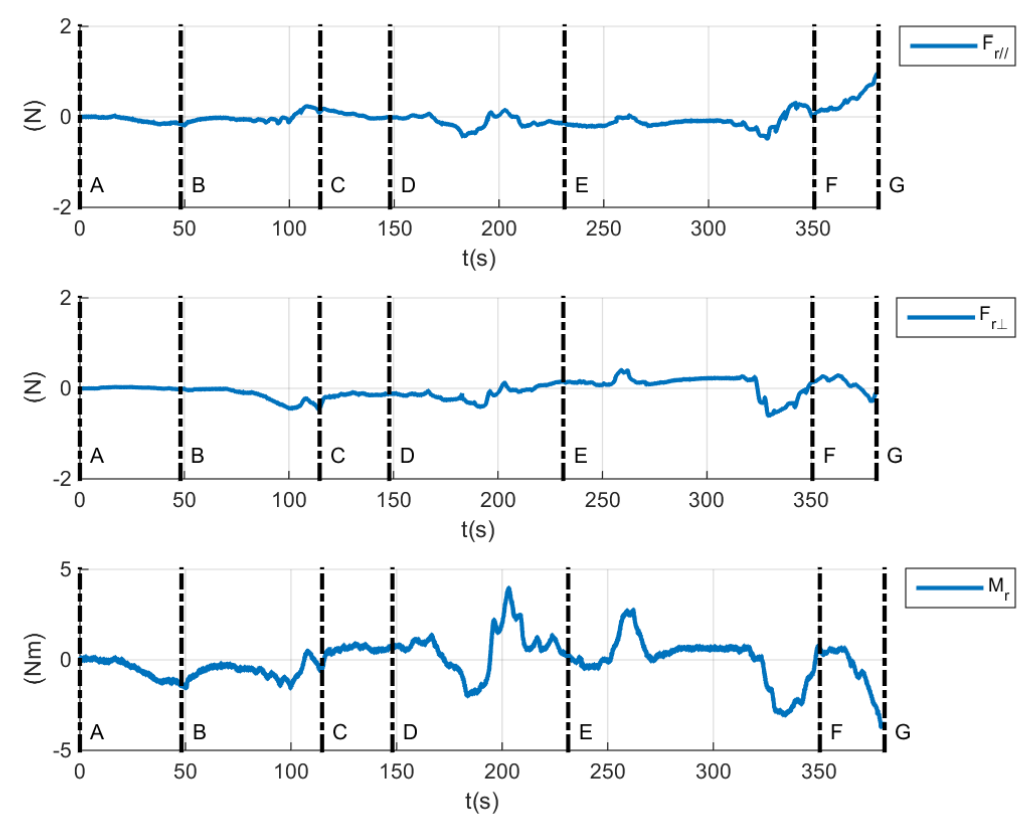

Figure 28: test 3: Time evolution of ${ }^{p} \mathbb{F}_{r}$.

\subsection{Test 4}

In this latter test, some removable walls have been added to the scene. In particular a holed wall is installed in parallel with one of the tank's walls (see figure 29). As in all the tests, the probe is initially in the pose $A$, and the operator starts by following a wall from $A$ to $B$, then rotates around a corner from $B$ to $C$, and translates along a second wall from $C$ to $D$. Between the poses $D$ and $E$ (figure 29(a)), the influence of the corner dominates while between $E$ and $F$, the operator perceives the influence of the removable wall, and finally, that of the hole. The point $E$ is the transitional point when shifting from one influence to the other. In contrast to the previous tests, this is very difficult to interpret the results by using simple models of electrokinetics. Indeed, the presence of the two walls and of a hole with sharp edges introduces multiple reflections and singularities (with point-effect) that are beyond analytical models in the field. Nonetheless, one can observe that the operator rotates correctly around the corner $(D \rightarrow E)$ and follows the removable wall $(E \rightarrow G)$ before being attracted by the hole beyond $G$. In this latter phase, the probe discovers the wall of the tank and attracts the operator in order to recover the equilibrium of the restoring force $F_{r \|}$ (see the top plot of figure 31 between $G$ and $H$ ). 


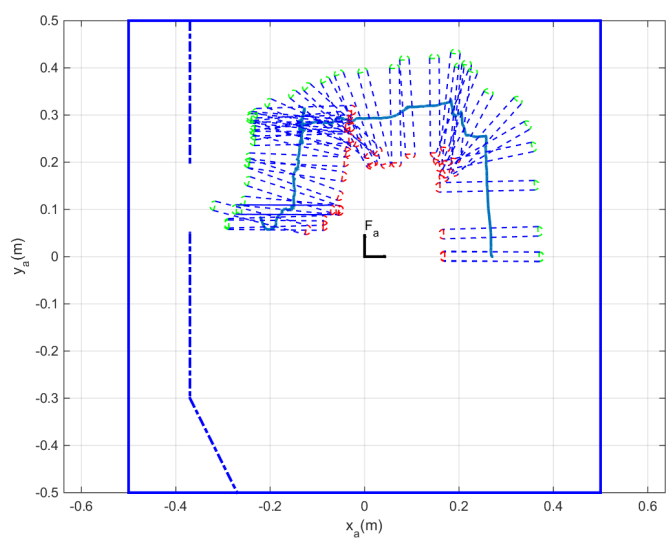

(a)

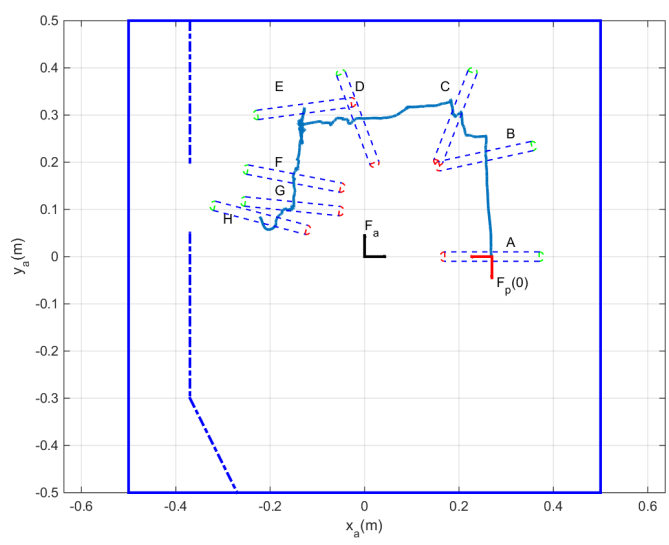

(b)

Figure 29: Test 4: Path of the probe in the slave space (a), with some distinguished poses (b).
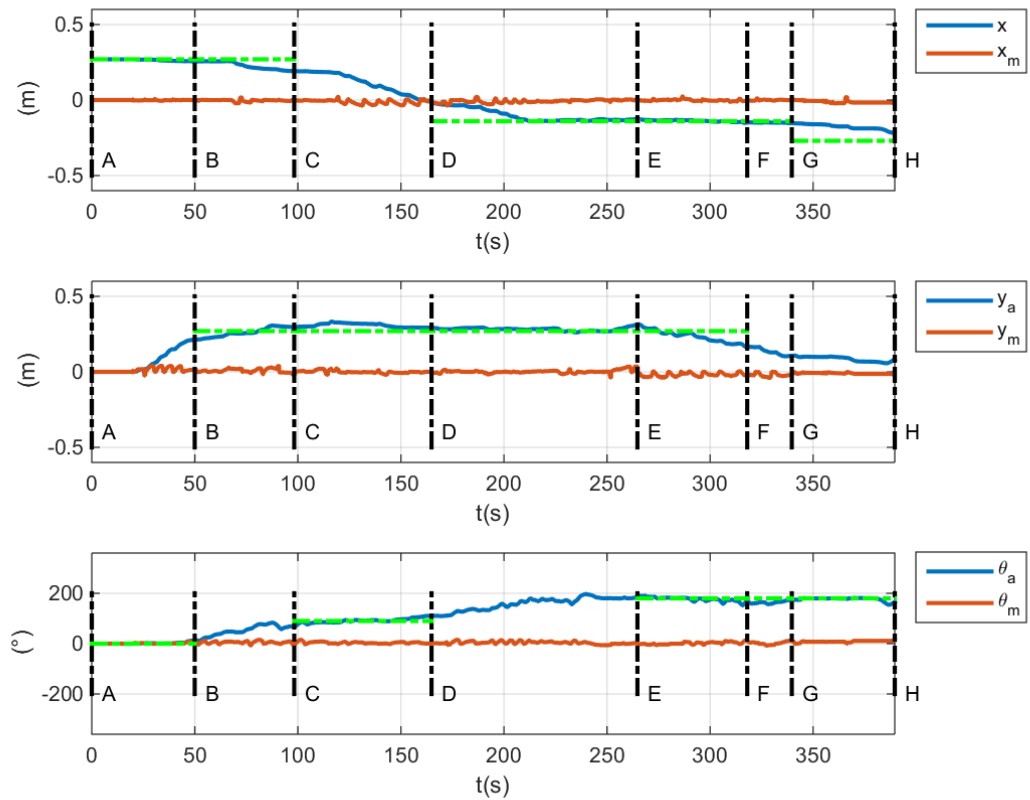

Figure 30: Test 4: Time evolution of $X_{m}$ and $X_{s}$. 

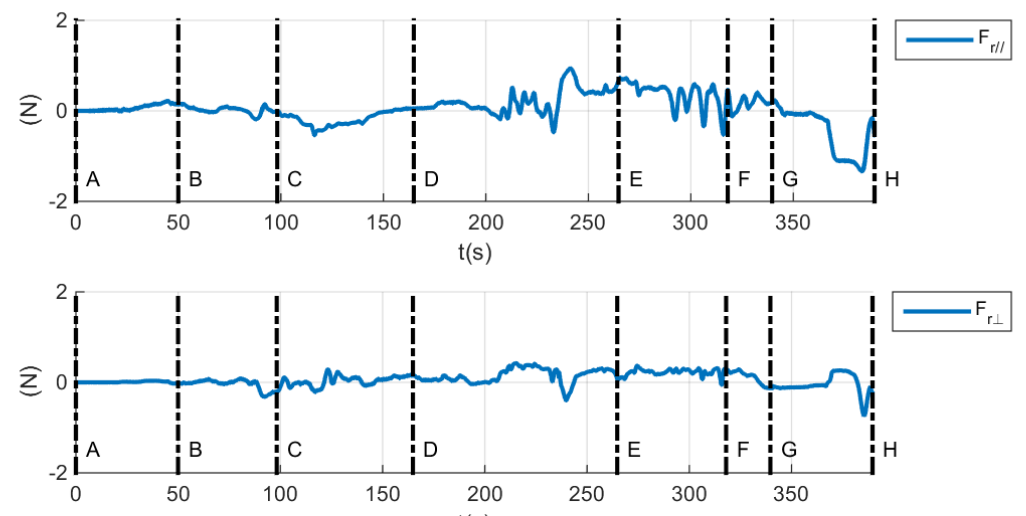

$\mathrm{t}(\mathrm{s})$

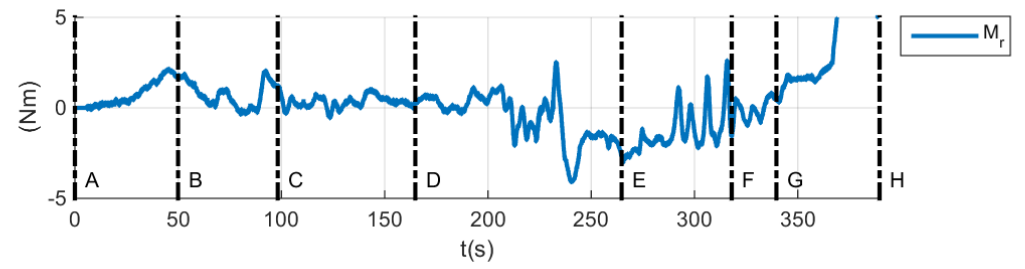

Figure 31: test 4: Time evolution of ${ }^{p} \mathbb{F}_{r}$.

\section{Conclusion}

In this article, we presented the first implementation of underwater artificial electric sense in a haptic feedback loop. To that aim, we used the haptic modality of this bio-inspired sense, to emulate some pre-touch forces able to assist an operator to feel and follow the boundaries of immersed objects. This issue remains challenging for underwater robotics especially in cluttered spaces with turbid waters, which are the natural working conditions of electric sense. The model of emulated force has been sought in the form of a restoring wrench exerted onto an active electric probe measuring the electric field reflected by the obstacles. To derive the model of the electric forces fed back to the operator through an haptic interface, we first exploited the currents measured by the probe to reconstruct the electric field along its body. Based on this reconstructed field, we were able to emulate a set of Coulomb forces and couples, and finally the expected model. Remarkably, this model can be interpreted as a three-dimensional extension of the attractiverepulsive Lennard-Johns force field modelling the atomic interactions in liquids and gas. Taking benefit from the properties of electrostatic interactions, this model is passive. This is a key property since, once coupled with a position-position tele-operating system, such a passive model guarantees the stability of the whole remote control loop. Finally, the feasibility of the concept has been proved through experiments including a first set of operational tests in different conditions. These results tend to confirm our theoretical predictions and show that artificial electric-sense could be useful for assisting other sensorial feedbacks for underwater tele-manipulation with no visibility. Beyond this proof of concept, this work opens further perspectives in electric field sensing for robotics, especially in air, where capacitive sensors could be used in the same way as the resistive sensing probe of this article. 


\section{References}

Aliaga, I., Rubio, A. \& Sanchez, E. (2004), 'Experimental quantitative comparison of different control architectures for master-slave teleoperation', IEEE Transactions on Control Systems Technology 12(1), 2-11.

Bai, Y., Snyder, J., Peshkin, M. \& MacIver, M. (2015), 'Finding and identifying simple objects underwater with active electrosense', The International Journal of Robotics Research .

Bai, Y., Snyder, J., Silverman, Y., Peshkin, M. \& MacIver, M. (2012), Sensing capacitance of underwater objects in bio-inspired electrosense, in '2012 IEEE/RSJ International Conference on Intelligent Robots and Systems (IROS)', pp. 1467-1472.

Boyer, F., Gossiaux, P., Jawad, B., Lebastard, V. \& Porez, M. (2012), 'Model for a sensor inspired by electric fish', IEEE Transactions on Robotics 28(2), 492-505.

Boyer, F., Lebastard, V., Chevallereau, C., Mintchev, S. \& Stefanini, C. (2015), 'Underwater navigation based on passive electric sense: New perspectives for underwater docking', The International Journal of Robotics Research 34(9), 1228-1250.

Boyer, F., Lebastard, V., Chevallereau, C. \& Servagent, N. (2013), 'Underwater reflex navigation in confined environment based on electric sense', IEEE Transactions on Robotics 29(4), 945-956.

Caputi, A., Aguilera, P., Carolina Pereira, A. \& Rodríguez-Cattáneo, A. (2013), 'On the haptic nature of the active electric sense of fish', Brain research 1536, 27-43.

URL: https://doi.org/10.1016/j.brainres.2013.05.028

Caputi, A., Budelli, R., Grant, K. \& Bell, C. (1998), 'The electric image in weakly electric fish: physical images of resistive objects in gnathonemus petersii.', Journal of experimental biology 201(14), 2115-2128.

Coiffet, P. \& Vertut, J. (1985), Teleoperation and Robotics Vol. $3 A$ and 3B, Kluwer Academic Publishers.

Colgate, J. \& Brown, J. (1994), Factors affecting the z-width of a haptic display, in '1994 IEEE International Conference on Robotics and Automation,', IEEE, pp. 3205-3210.

Fang, S., Peshkin, M. \& MacIver, M. (2016), 'Human-in-the-loop active electrosense', Bioinspiration \& Biomimetics 12, 014001.

URL: http://iopscience.iop.org/article/10.1088/1748-3190/12/1/014001/meta

Geffard, F., Garrec, P., Piolain, G., Brudieu, M.-A., Thro, J.-F., Coudray, A. \& Lelann, E. (2012), 'Tao2000 v2 computer-assisted force feedback telemanipulators used as maintenance and production tools at the areva nc-la hague fuel recycling plant', Journal of Field Robotics 29(1), 161-174.

Gosselin, F., Martins, J. P., Bidard, C., Andriot, C. \& Brisset, J. (2005), Design of a new parallel haptic device for desktop applications, in 'First Joint Eurohaptics Conference and Symposium on Haptic Interfaces for Virtual Environment and Teleoperator Systems. World Haptics Conference', pp. 189-194. 
Hannaford, B. (1989), 'A design framework for teleoperators with kinesthetic feedback', IEEE transactions on Robotics and Automation 5(4), 426-434.

Hannaford, B. \& Ryu, J.-H. (2002), 'Time-domain passivity control of haptic interfaces', IEEE Transactions on Robotics and Automation 18(1), 1-10.

Hirschfelder, J., Curtiss, C. \& Robert, B. (1966), Molecular Theory of Gases and Liquids, John Wiley and Sons.

Hogan, N. (1985), 'Impedance control: An approach to manipulation: Parts i,ii,iii', Journal of Dynamic Systems, Measurement, and Control 107, 1-24.

Hogan, N. (1989), Controlling impedance at the man/machine interface, in 'Proceedings, 1989 International Conference on Robotics and Automation', Vol. 3, pp. 1626-1631.

Hopkins, C. (2005), Passive electrolocation and the sensory guidance of oriented behavior, in 'Electroreception', Encyclopedia of Perception, Springer, pp. 264-289.

Jackson, J. D. (1999), Classical electrodynamics, 3rd edn, Wiley, New York.

Kalmijn, A. (1971), 'The Electric Sense of Sharks and Rays', Journal of Experimental Biology 55(2), 371-383.

URL: http://jeb.biologists.org/content/55/2/371

Lanneau, S., Boyer, F., Lebastard, V. \& Bazeille, S. (2017), 'Model based estimation of ellipsoidal object using artificial electric sense', The International Journal of Robotics Research 36(9), 1022-1041.

Lebastard, V., Boyer, F. \& Lanneau, S. (2016), 'Reactive underwater object inspection based on artificial electric sense', Bioinspiration \& Biomimetics 11(4), 45003-45020.

Lebastard, V., Chevallereau, C., Girin, A., Servagent, N., Gossiaux, P.-B. \& Boyer, F. (2013), 'Environment reconstruction and navigation with electric sense based on a kalman filter', The International Journal of Robotics Research 32(2), 172-188.

Lissmann, H. \& Machin, K. (1958), 'The mechanism of object location in gymnarchus niloticus and similar fish', Journal of Experimental Biology 35(2), 451-486.

URL: http://jeb.biologists.org/content/35/2/451

Maalouf, D., Creuze, V. \& Chemori, A. (2012), State feedback control of an underwater vehicle for wall following, in '2012 20th Mediterranean Conference on Control Automation (MED)', pp. $542-547$.

Mitra, P. \& Niemeyer, G. (2004), Dynamic proxy objects in haptic simulations, in 'IEEE Conference on Robotics, Automation and Mechatronics, 2004.', Vol. 2, pp. 1054-1059 vol.2.

Murray, R., Li, Z. \& Sastry, S. (1994), A mathematical introduction to robotic manipulation, CRC press.

Salcudean, S., Wong, N. \& Hollis, R. (1995), 'Design and control of a force-re ecting teleoperation system with magnetically levitated master and wrist', IEEE Transactions on Robotics and Automation pp. 844-858. 
Salisbury, J. (1980), Active stiffness control of a manipulator in cartesian coordinates, in '1980 19th IEEE Conference on Decision and Control including the Symposium on Adaptive Processes', pp. 95-100.

Servagent, N., Jawad, B., Bouvier, S., Boyer, F., Girin, A., Gomez, F., Lebastard, V., Stefanini, C. \& Gossiaux, P.-B. (2013), 'Electrolocation sensors in conducting water bio-inspired by electric fish', Sensors Journal, IEEE 13(5), 1865-1882.

Silverman, Y., Snyder, J., Bai, Y. \& MacIver, M. (2012), Location and orientation estimation with an electrosense robot, in 'Workshop on IEEE/RSJ International Conference on Intelligent Robots and Systems (IROS),', pp. 4218-4223.

Solberg, J., Lynch, K. \& MacIver, M. (2008), 'Active electrolocation for underwater target localization', The International Journal of Robotics Research 27(5), 529-548.

Von der Emde, G. \& Bleckmann, H. (1998), 'Finding food: senses involved in foraging for insect larvae in the electric fish gnathonemus petersii', Journal of Experimental Biology 201(7), 969980. 